\title{
REVIEW
}

Received 10 Jul 2014 | Accepted 21 Nov 2014 | Published 20 Jan 2015

DOI: $10.1038 /$ ncomms 6933

\section{Using carbon dioxide as a building block in organic synthesis}

\author{
Qiang Liu', Lipeng Wu', Ralf Jackstell ${ }^{1} \&$ Matthias Beller ${ }^{1}$
}

Carbon dioxide exits in the atmosphere and is produced by the combustion of fossil fuels, the fermentation of sugars and the respiration of all living organisms. An active goal in organic synthesis is to take this carbon-trapped in a waste product-and re-use it to build useful chemicals. Recent advances in organometallic chemistry and catalysis provide effective means for the chemical transformation of $\mathrm{CO}_{2}$ and its incorporation into synthetic organic molecules under mild conditions. Such a use of carbon dioxide as a renewable one-carbon (C1) building block in organic synthesis could contribute to a more sustainable use of resources.

\begin{abstract}
A more sensible resource management is the prerequisite for the sustainable development of future generations. However, when dealing with the feedstock of the chemical industry, the level of sustainability is still far from satisfactory. Until now, the vast majority of carbon resources are based on crude oil, natural gas and coal. In addition to biomass, $\mathrm{CO}_{2}$ offers the possibility to create a renewable carbon economy. Since pre-industrial times, the amount of $\mathrm{CO}_{2}$ has steadily increased and nowadays $\mathrm{CO}_{2}$ is a component of greenhouse gases, which are primarily responsible for the rise in atmospheric temperature and probably abnormal changes in the global climate. This increase in $\mathrm{CO}_{2}$ concentration is largely due to the combustion of fossil fuels, which are required to meet the world's energy demand ${ }^{1}$. Obviously, there is an urgent need to control $\mathrm{CO}_{2}$ emissions and develop efficient carbon capture systems. Although the extensive use of carbon dioxide for chemical production cannot solve this problem alone, $\mathrm{CO}_{2}$ is a useful one-carbon $(\mathrm{C} 1)$ building block in organic synthesis due to its abundance, availability, nontoxicity and recyclability. As a result, valorization of $\mathrm{CO}_{2}$ is currently receiving considerable and ever increasing attention by the scientific community ${ }^{2-4}$. However, activation and utilization of $\mathrm{CO}_{2}$ is still problematic due to the fact that it is the most oxidized form of carbon, which is also thermodynamically stable and/or kinetically inert in certain desired transformations. Consequently, most of the known studies used highly reactive substrates and/or severe reaction conditions to activate $\mathrm{CO}_{2}$, limiting the application of such methods. In particular, the catalytic coupling of $\mathrm{CO}_{2}$ with energy-rich substrates, such as epoxides and aziridines, to generate polycarbonates/polycarbamates and/or cyclic carbonates/carbamates has drawn significant attention over the past decades. To create $\mathrm{C}-\mathrm{C}$ bonds with $\mathrm{CO}_{2}$, the use of carbon nucleophiles is specifically limited to strong nucleophilic organolithiums and Grignard reagents, as well as phenolates.
\end{abstract}

\footnotetext{
${ }^{1}$ Leibniz-Institut für Katalyse an der Universität Rostock, Albert-Einstein-Strasse 29a, 18059 Rostock, Germany. Correspondence should be addressed to M.B. (email: matthias.beller@catalysis.de).
} 
Along with the rapid development of organometallic chemistry and catalysis (see Box 1), various types of efficient $\mathrm{CO}_{2}$ transformations in organic synthesis have been discovered in the past decades, greatly improving its efficiency and applicability.

This review will describe the most recent advances made in the area of $\mathrm{CO}_{2}$ valorization-turning $\mathrm{CO}_{2}$ into a useful chemical feedstock - under mild conditions. A special focus is given on the reaction modes for the $\mathrm{CO}_{2}$ activation and its application as $\mathrm{C} 1$ building block in organic synthesis. While classical methods for $\mathrm{CO}_{2}$ hydrogenation have been reviewed elsewhere and will not be included here ${ }^{5-7}$, the following subjects will be addressed: (1) novel transformations using carbon dioxide (briefly summarized in Fig. 1); (2) different reaction modes for $\mathrm{CO}_{2}$ activation (main focus of this review); and (3) potential new applications of $\mathrm{CO}_{2}$ valorization.

\section{Activation modes for catalytic valorization of $\mathrm{CO}_{2}$}

$\mathrm{CO}_{2}$ is the most stable form of oxidized carbon compounds. Nevertheless, it reacts easily with strong nucleophiles to form new $\mathrm{C}-\mathrm{C}$ or $\mathrm{C}-\mathrm{H}$ bonds. However, to use carbon dioxide in a more benign and practical manner, efficient transformations with lessactivated substrates under mild conditions have to be developed. Obviously, reactions of $\mathrm{CO}_{2}$ that require a high-energy input are not benign because in general this energy leads also to formation of $\mathrm{CO}_{2}$.

Cooperative effects for $\mathrm{CO}_{2}$ activation. To achieve low-energydemanding synthetic protocols using $\mathrm{CO}_{2}$ as $\mathrm{C} 1$ building block, efficient interactions with the specific substrate is required. In this respect, cooperative activation of carbon dioxide with such substrates often in the presence of a suitable catalyst is of fundamental importance. Among the $\mathrm{CO}_{2}$ functionalization methodologies, traditional examples that make use of cooperative effects are the catalytic valorization of energy-rich substrates (for example, strong nucleophilic organometallic reagents, epoxides, aziridines and so on). They provide a straightforward way for the thermodynamic driving force.
One of the more successful processes for $\mathrm{CO}_{2}$ utilization for material synthesis is the catalytic productions of cyclic carbonates and polycarbonates from epoxides ${ }^{8}$, which has been industrialized as well ${ }^{9}$. Nowadays, cyclic carbonates are widely used as organic synthetic intermediates, aprotic polar solvents, precursors for biomedical applications and raw materials for engineering plastics. Although this $100 \%$ atom economical reaction has been commercialized for the synthesis of ethylene and propylene carbonates for over 50 years ${ }^{10}$, recently a variety of highly selective and reactive catalysts were developed. Representative systems are summarized in Fig. 2 (ref. 11). Notably, in 1983, Inoue and co-workers described in their seminal work that a (TPP)AlOMe complex can trap carbon dioxide readily and reversibly at room temperature. The trapped carbon dioxide is active enough to react with epoxide at ambient conditions to afford the corresponding cyclic carbonates ${ }^{12}$. Since then, various metalloprophyrin catalysts were studied in this transformation. For example, in 2007, Jing et al. ${ }^{13}$ reported a $\mathrm{Co}(\mathrm{TPP})(\mathrm{OAc}) / \mathrm{PTAT}$ (phenyltrimethylammonium tribromide) catalyst system to achieve this $\mathrm{CO}_{2}$ fixation at room temperature and low pressure. More recently, Sakai et al. developed a highly active bifunctional porphyrin catalyst, which combines an organocatalytic group (quaternary ammonium bromide) and a metal ion $\left(\mathrm{Mg}^{\mathrm{II}}\right)$. It shows a high turnover number $(\mathrm{TON}=103,000)$ for the synthesis of cyclic carbonates from $\mathrm{CO}_{2}$ and epoxides under solvent-free conditions ${ }^{14}$. Related salenbased complexes represent another kind of effective catalysts for the electrophilic addition of $\mathrm{CO}_{2}$ to epoxides. Already in 2001, air stable and commercially available $\mathrm{Cr}$ (III) salen complexes were presented to be highly efficient catalysts in the coupling of carbon dioxide with epoxides at mild temperatures and pressures by the group of Nguyen ${ }^{15}$. While most of the work in this area made use of pressurized carbon dioxide, Kleij and co-workers ${ }^{16}$ showed that inexpensive and structurally simple $\mathrm{Zn}$ (salphen) catalysts can be employed as efficient catalysts for the synthesis of cyclic carbonates from $\mathrm{CO}_{2}$ and terminal epoxides under mild conditions (2 bar $\mathrm{CO}_{2}$ ). Noteworthy, the group of North ${ }^{17}$ communicated the formation of cyclic carbonates even at $25^{\circ} \mathrm{C}$

\section{Box 1 | Coordination modes of $\mathrm{CO}_{2}$ with transition metals.}

A key issue for valorization of $\mathrm{CO}_{2}$ to valuable chemical intermediates and further on to consumer products relies on efficient catalysts. As a basis, an insightful understanding of the detailed organometallic chemistry of $\mathrm{CO}_{2}$ is of great importance for the further design of the catalytic processes. In fact the chelating mode between transition metal centres and $\mathrm{CO}_{2}$ has been investigated intensively via stoichiometric experimental studies ${ }^{46,47}$
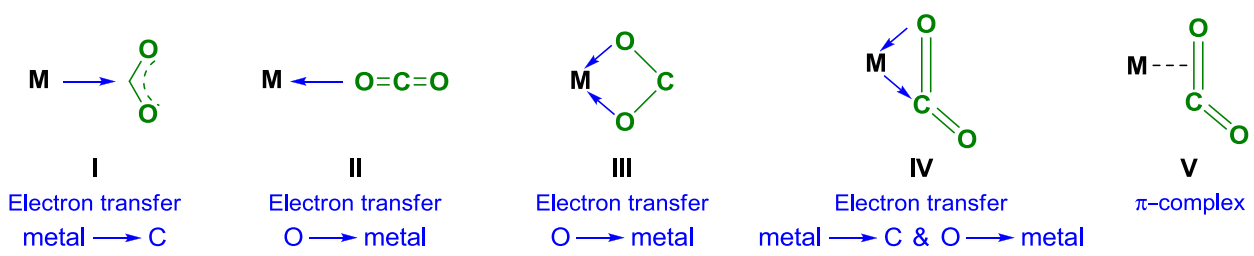

As shown above, $\mathrm{CO}_{2}$ has multiple reactive sites: the carbon atom is an electrophilic Lewis acid centre and the oxygen atoms act as weak nucleophilic Lewis base. In its ground state, carbon dioxide possesses two equivalent $\mathrm{C}-\mathrm{O}$ bonds that could both coordinate to a transition metal centre. As a result, a series of transition metal $\mathrm{CO}_{2}$ complexes are known. If a metal centre reacts with one molecule of $\mathrm{CO}_{2}$, there are five different chelating modes possible. More specifically, complex I with an $\mathrm{M}-\mathrm{C}$ bond is sometimes termed as a metallacarboxylate. Electron-rich metal centres are more feasible to form these types of complexes via electron transfer from metal centre to carbon atom. Adduct II (end-on type) seems less plausible due to the weak interaction between the lone pair of only one oxygen atom and the metal centre. Meanwhile, complex III should be more stable, since $\mathrm{CO}_{2}$ acts as a bidentate ligand with two oxygen atoms. In this case, a more electron-deficient metal centre is favoured for the electron transfer from oxygen atom to transition metal. A combination of the above two mentioned electron transfer processed affords the three-membered metallacycle complex IV. Moreover, the side-on-bonding $\pi$-complex $\mathbf{V}$ can also be formed in a similar spatial arrangement of atoms through the coordination of the $\mathrm{C}$-O double bond to the central metal.

On the basis of these different coordination and activation modes, well-designed transition metal complex catalysts are able to promote the reactivity and also control the selectivity of $\mathrm{CO}_{2}$ fixation reactions in the organic synthesis. This promising research area still has many novelties to be discovered. 


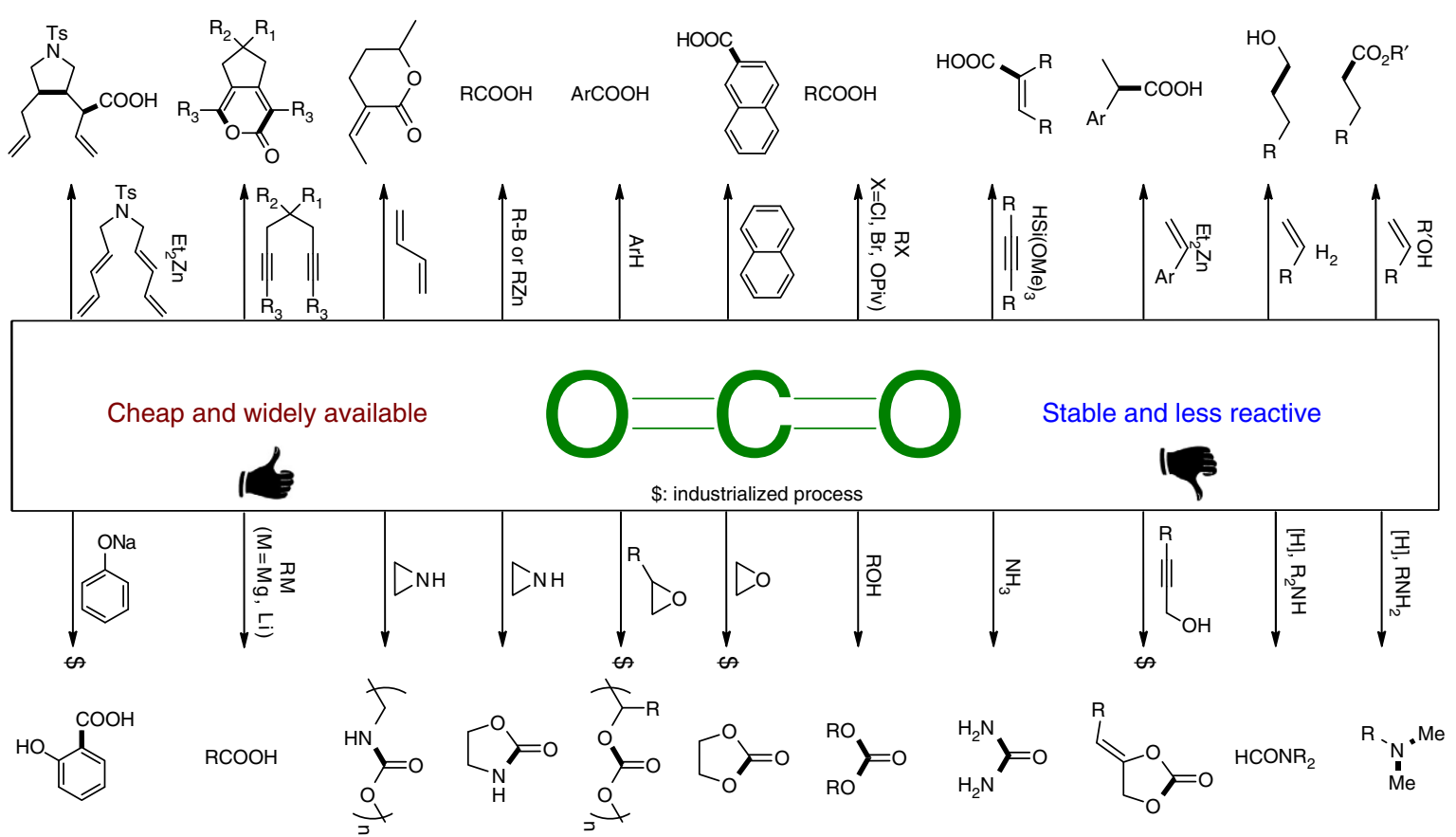

Figure 1 | Representative examples using $\mathbf{C O}_{2}$ as $\mathbf{C 1}$ building block in organic synthesis. Along with the rapid development of organometallic chemistry and catalysis, various types of efficient $\mathrm{CO}_{2}$ transformations were have been discovered in the past decades. However, in general, the substrates' scope and efficiency of these reactions are still limited due to the requirement of reactive agents for $\mathrm{CO}_{2}$ activation. As a result, only a few processes have been industrialized until now (marked by $\$$ ).

and 1 bar $\mathrm{CO}_{2}$ using bimetallic $\mathrm{Al}^{\mathrm{III}}$-salen complexes. More recently, the Kleij group ${ }^{18}$ presented an easily accessible amino triphenolate complex based on $\mathrm{Al}^{\mathrm{III}}$ metal centre. This complex is demonstrated to be a highly active and versatile catalyst for organic carbonate formation. Remarkably, initial turnover frequencies as high as 36,000 per hour during the cycloaddition of $\mathrm{CO}_{2}$ to epoxides were obtained with TONs exceeding 100,000. Except for the above-mentioned sophisticated metal complexes, simple onium salts can also acts as efficient catalysts. For instance, a combination of $\mathrm{ZnCl}_{2}$ and $[\mathrm{Bmim}] \mathrm{Br}$ reported by Xia's group ${ }^{19}$ is a really practical, highly efficient and environmentally benign catalyst system for the chemical fixation of $\mathrm{CO}_{2}$ with epoxides. Finally, it is worth mentioning that Jiang, Han and co-workers ${ }^{20}$ in 2011 found that cellulose/KI was a very active, selective and recyclable catalyst for this reaction without the assistance of any transition metal catalyst.

The direct polymerization of epoxides with $\mathrm{CO}_{2}$ to produce polycarbonates has been accomplished too. In this respect, the contributions from Coates and co-workers ${ }^{21,22}$ described very active zinc $\beta$-diiminate catalysts for the synthesis of monodispersed, highly alternating carbon dioxide/epoxide copolymers with high molecular weight. In addition, the group of Williams ${ }^{23}$ demonstrated a zinc-based macrocyclic bimetallic catalyst for epoxide/ $\mathrm{CO}_{2}$ copolymerization, which shows remarkable activity at only 1 bar of $\mathrm{CO}_{2}$. Very recently, iron-corrole complexes were synthesized by Nozaki and co-workers ${ }^{24}$ to copolymerize epoxides with $\mathrm{CO}_{2}$, providing the first example of iron-catalysed $\mathrm{PO}$ (propylene oxide) $/ \mathrm{CO}_{2}$ and GPE (glycidyl phenyl ether)/ $\mathrm{CO}_{2}$ copolymerization. The same group also demonstrated the stereoselective alternating copolymerization of cyclohexene oxide and $\mathrm{CO}_{2}$ with dimeric zinc complexes for the first time ${ }^{25}$. Besides, improved catalysts are still desirable to better control the properties of the resulting materials ${ }^{26}$. Similarly, many efforts are also devoted to designing catalysts able to promote the reaction of $\mathrm{CO}_{2}$ with less-reactive oxiranes, which could also provide novel materials ${ }^{27}$.
Apart from the utilization of energy-rich substrates (for example, epoxides), catalytic reductive formylation and methylation of amines represent important modern examples for cooperative effects in the activation of $\mathrm{CO}_{2}$. Here the activation energy for reductive deoxygenation of $\mathrm{CO}_{2}$ is significantly decreased by the coordination of amines to $\mathrm{CO}_{2}$ and the formation of $\mathrm{N}-\mathrm{C}$ bonds (in a carbamate or urethane intermediate), which thus facilitates the reduction of $\mathrm{CO}_{2}$. Likely, the other advantage of amines is to increase $\mathrm{CO}_{2}$ concentration in the condensed phase ${ }^{28}$. These transformations represent diagonal approaches to the chemical recycling of $\mathrm{CO}_{2}$, which combine both reduction of $\mathrm{CO}_{2}$ and $\mathrm{C}-\mathrm{N}$ bond formation processes $(\text { Fig. } 3)^{29}$. More specifically, Cantat and co-workers reported an unprecedented organocatalytic synthesis of formamides from $\mathrm{CO}_{2}$. Mechanistic studies uncovered the synergistic effect of the organocatalyst TBD (triazabicyclodecene) and amine substrates in the promotion of $\mathrm{CO}_{2}$ activation (Fig. 3, equation a) ${ }^{30}$. Shortly afterwards, a highly active $\mathrm{N}$-heterocyclic carbene organocatalyst was designed by the same group for the formylation of $\mathrm{N}-\mathrm{H}$ bonds in various amines and heterocycles under very mild conditions using $\mathrm{CO}_{2}$ and polymethylhydrosiloxane (PMHS; Fig. 3, equation $b)^{31}$.

Meanwhile, the methylation of amines via six-electron reduction of $\mathrm{CO}_{2}$ remains unknown until 2013. The market value of methylamines such as $\mathrm{MeNH}_{2}, \mathrm{Me}_{2} \mathrm{NH}$ and $\mathrm{Me}_{3} \mathrm{~N}$ exceeds 4,000 Euro per ton. Therefore, the reductive methylation of amines with $\mathrm{CO}_{2}$ can create additional value. In early 2013, our group developed a $\mathrm{Ru}$ catalyst system that was able to convert carbon dioxide and amines into various kinds of $\mathrm{N}$-methylated products in the presence of hydrosilanes $\left(\mathrm{PhSiH}_{3}\right)$. Notably, diverse functional groups were well tolerated under these conditions (Fig. 3, equation $\mathrm{c}^{32}$. In the same year, Cantat et al. ${ }^{33}$ reported zinc catalysts able to perform the methylation of amines with $\mathrm{CO}_{2}$ and hydrosilanes at low pressure. Furthermore, the selective reduction of ureas was possible under similar reaction conditions. Although some silanes are considered to be 


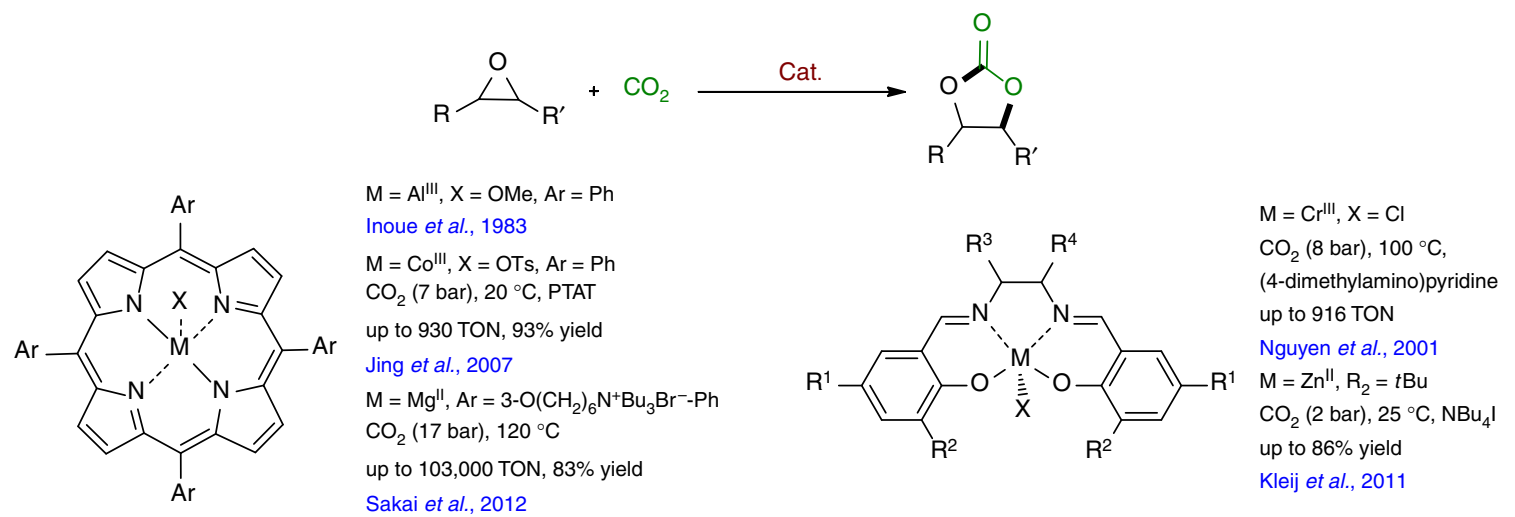

Metalloporphyrin catalyst

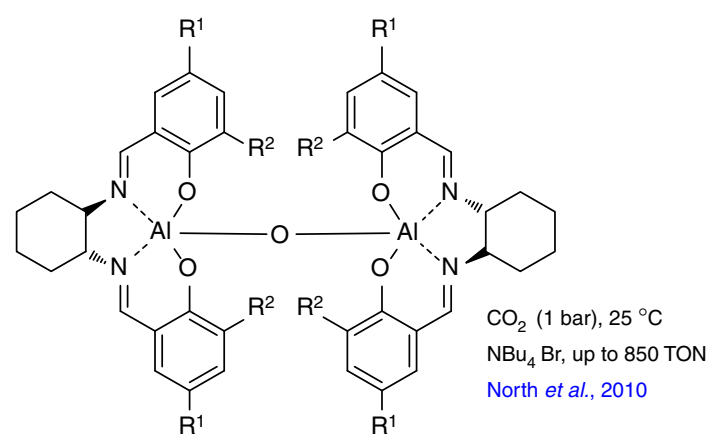

Bimetallic aluminium(salen) catalyst<smiles>[Z10][13CH2][13CH2]CCCn1cc[n+](C)c1</smiles>

$\mathrm{CO}_{2}(15 \mathrm{bar}), 100^{\circ} \mathrm{C}, \mathrm{KI}$

Up to 5,580 TON, 5,580 per hour TOF

Xia et al., 2004

Onium salts catalyst
Salen catalyst

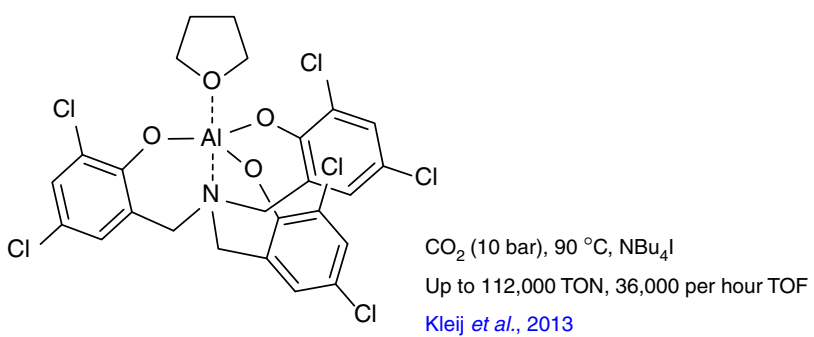

Amino triphenolate aluminium catalyst

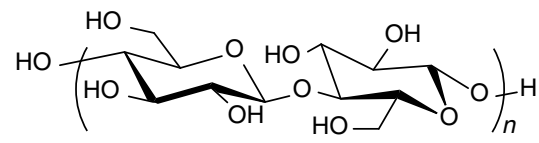

$\mathrm{CO}_{2}(20 \mathrm{bar}), 110^{\circ} \mathrm{C}, \mathrm{KI}$

Up to $99 \%$ yield

Jiang and Han et al., 2011

Cellulose catalyst

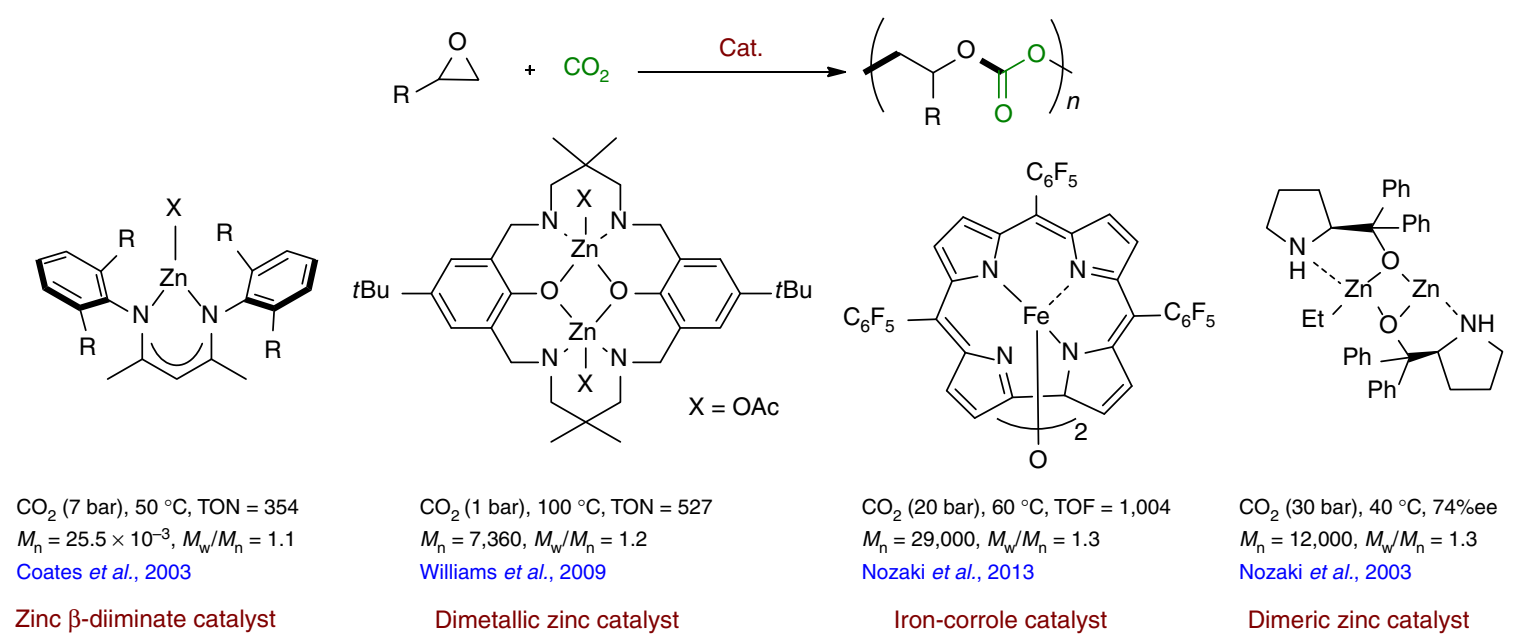

Figure 2 | Established catalysts and reaction conditions for the catalytic productions of cyclic carbonate and polycarbonate from oxiranes using $\mathrm{CO}_{2}$ as the feedstock. Examples include metalloporphyrin, salen, metal-corrole, dimetallic as well as metal-free catalysts.

waste products of the silicone industry, all these methodologies are limited by the accessibility of the hydrosilanes and an additional workup step to remove siloxane by-products. Obviously, catalytic methylations using $\mathrm{CO}_{2}$ and $\mathrm{H}_{2}$ represent a greener method with $\mathrm{H}_{2} \mathrm{O}$ as the only by-product. In this respect, two research groups independently reported similar catalytic systems shortly afterwards to convert amines into methylamines in the presence of $\mathrm{CO}_{2}$ and $\mathrm{H}_{2}$. On one hand, Klankermayer and co-workers $^{34}$ presented the use of a molecularly defined ruthenium complex $[\mathrm{Ru}($ triphos $)(\mathrm{tmm})]$ together with readily available organic acids as co-catalysts to afford the methylation of aryl amines in good yields (Fig. 3, equation d). Meanwhile, the 
a

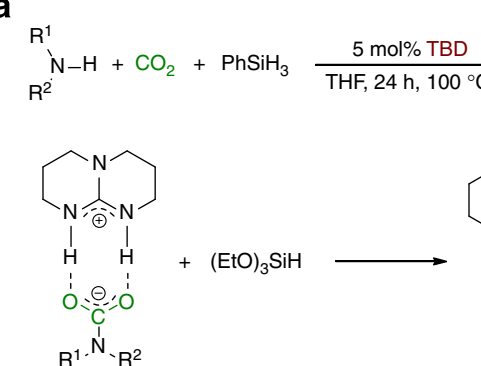

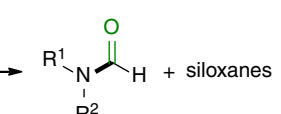

$24-100 \%, 7$ examples

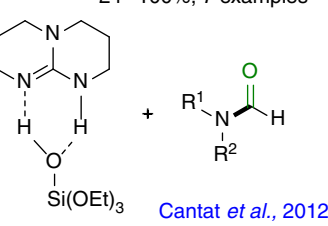

$\mathrm{R}^{1}$

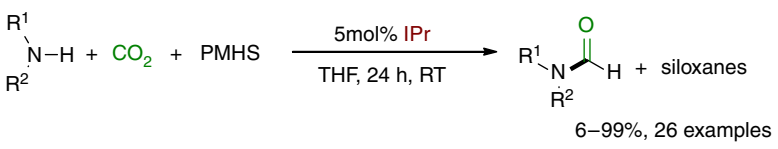

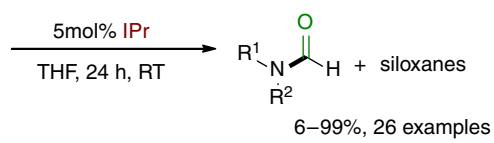

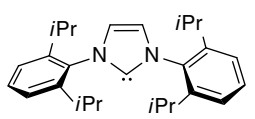

$\operatorname{IPr}$

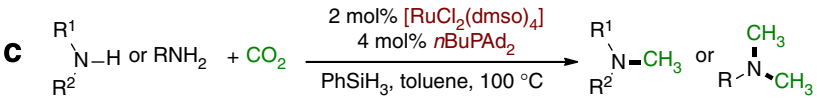

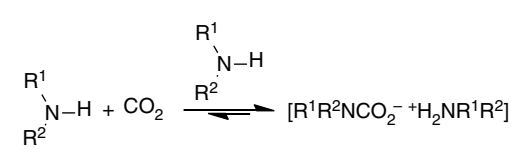

$$
\begin{aligned}
& {[\mathrm{Ru}] /[\mathrm{H}] \downarrow-\mathrm{H}_{2} \mathrm{O}} \\
& \underset{\mathrm{R}^{2}}{\mathrm{R}-\mathrm{O}} \\
& \frac{[\mathrm{Ru}][\mathrm{H}]}{-\mathrm{H}_{2} \mathrm{O}} \\
& \text { 63-99\%, } 25 \text { examples }
\end{aligned}
$$

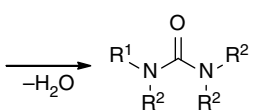

$$
\begin{aligned}
& {[\mathrm{Ru}][\mathrm{H}] \underbrace{\mathrm{R}^{1} \mathrm{~N}-\mathrm{H}}_{\mathrm{R}^{2}}} \\
& \mathrm{R}^{1} \\
& \mathrm{~N}-\mathrm{CH}_{3}
\end{aligned}
$$

Beller et al., 2013

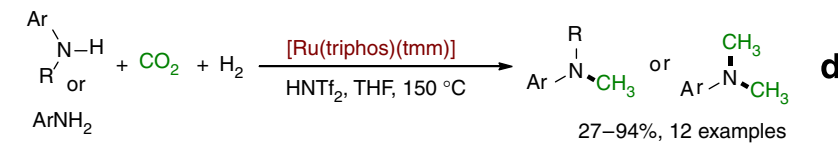
$\mathrm{Ph}_{2} \mathrm{P} \overbrace{\mathrm{PPh}_{2}}$ Triphos

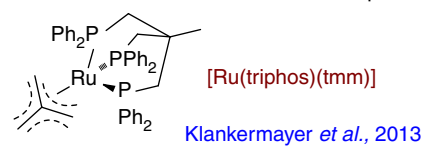

$$
\begin{aligned}
& 1 \mathrm{~mol} \% \mathrm{Ru}(\mathrm{acac})_{3}
\end{aligned}
$$

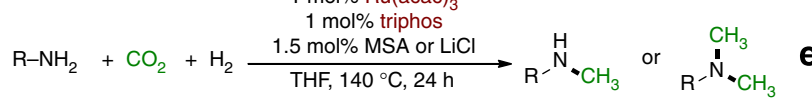

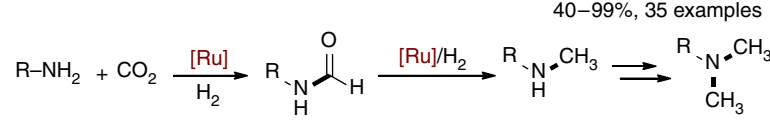

Figure 3 | Catalytic reductive formylation and methylation of amines with $\mathbf{C O}_{\mathbf{2}}$ as $\mathbf{C 1}$ source. (a,b) Organocatalytic formylation of amines with $\mathrm{CO}_{2}$ using silanes as the reductants. (c) Ru-catalysed methylation of amines with $\mathrm{CO}_{2}$ using silanes as the reductants. (d,e) Ru-catalysed methylation of amines with $\mathrm{CO}_{2}$ using $\mathrm{H}_{2}$ as the reductant.

group of Beller demonstrated the efficient $N$-methylation of both aromatic and aliphatic amines using $\mathrm{CO}_{2} / \mathrm{H}_{2}$ as the methylation reagent. Applying an in situ combination of $\mathrm{Ru}(\mathrm{acac})_{3}$, triphos and either acid additives or $\mathrm{LiCl}$, the desired methylated amines were obtained with high efficiency. Notably, selective monomethylation of primary amines as well as the methylation of more challenging aliphatic amines proceeded smoothly under these conditions (Fig. 3, equation e) ${ }^{35}$. Interestingly, Shi et al. ${ }^{36}$ described in 2014 heterogeneous copper catalysts for the same transformation with $\mathrm{CO}_{2}$ and $\mathrm{H}_{2}$. In general, formamides are the common reaction intermediates for the above-mentioned methylation reactions. Again, this indicates the advantageous activation effect of amine substrates for the $\mathrm{CO}_{2}$ reduction step.

$\mathrm{CO}_{2}$ fixation via oxidative cyclometallation. Pioneered by the synthesis and isolation of Aresta's complex $\left[\mathrm{Ni}\left(\mathrm{CO}_{2}\right)\left(\mathrm{PCy}_{3}\right)_{2}\right]$ in 1975 (ref. 37), the development of low-valent metal (nickel or palladium) complexes that mediate the oxidative couplings of $\mathrm{CO}_{2}$ with various unsaturated hydrocarbons provides a facile approach to overcome the obstacles of $\mathrm{CO}_{2}$ fixation. While it permitted the use of $\mathrm{CO}_{2}$ under atmospheric pressure, stoichiometric amounts of metal were necessary to generate various metallacycle complexes via the oxidative cyclometallation of $\mathrm{Ni}(0)$ complexes with $\mathrm{CO}_{2}$ and olefins or alkynes (Fig. 4, equations $\mathrm{a}$ and $\mathrm{b})^{38,39}$. The nickel-mediated stoichiometric fixation of carbon dioxide with alkenes ${ }^{40}$ has been known for over 20 years largely due to the works from Hoberg and co-workers. However, the limited substrate scope and/or relatively harsh conditions still remain concerns for this $\mathrm{CO}_{2}$ activation strategy. Meanwhile, only a few catalytic systems based on this strategy have been developed. In 2002, Louie et al. ${ }^{41}$ communicated an efficient nickel-catalysed $[2+2+2]$ cycloaddition of $\mathrm{CO}_{2}$ and diynes in the presence of catalytic amounts of $\mathrm{Ni}(0)$ and specific carbene ligands (Fig. 4, equation c). In the same year, the group of Mori ${ }^{42,43}$ developed a nickel-catalysed regio- and stereoselective ring-closing carboxylation of bis-1,3-dienes, which proceeds via insertion of $\mathrm{CO}_{2}$ into a bis- $\pi$-allylnickel intermediate followed by a transmetalation process of the resulting cyclic nickel carboxylate with a sensitive dialkylzinc reagent (Fig. 4, equation d). Except for nickel-catalysed cyclization reactions, the Pd-catalysed telomerization of 1,3-butadiene with $\mathrm{CO}_{2}$ is one of the first successful examples of the catalytic formation of a new C-C bond between $\mathrm{CO}_{2}$ and an organic compound. Thirty years ago, Behr et al. found that the cyclization product is selectively produced employing $\mathrm{Pd}(\mathrm{acac})_{3}$ and $\mathrm{P} \mathrm{Pr}_{3}$ as the catalyst. The resulting $\delta$-lactone is formed via an allylic palladium carboxylate complex as a key intermediate (Fig. 4, equation e ${ }^{44}$. Generally, the $\mathrm{CO}_{2}$ transformations via oxidative cyclometallation pathways are restricted to substrates with extensive $\pi$ systems (for example, dienes and diynes). Interestingly, in 2008, the group of Dong discovered that Aresta's complex catalysed the cross-coupling of organozinc reagents with $\mathrm{CO}_{2}$. By replacing nickel catalysts with palladium, the latter complexes were found to be catalytically active as well for the reaction of aryl zinc bromides (Fig. 4, equation $\mathrm{f}$ ). The proposed mechanism consisted of an oxidative addition of $\mathrm{Ni}(0)$ to $\mathrm{CO}_{2}$ to generate Aresta's complex; transmetalation with the organozinc reagent and reductive elimination affording the corresponding zinc carboxylate that was finally hydrolysed by acidic workup (Fig. 4, equation $\mathrm{g})^{45}$.

Carboxylation reactions via $\mathrm{CO}_{2}$ insertion into $\mathrm{C}-\mathrm{M}$ bonds. The formation of a thermodynamically and kinetically stable C-C bond is the most desirable form of $\mathrm{CO}_{2}$ fixation. Since carbon 


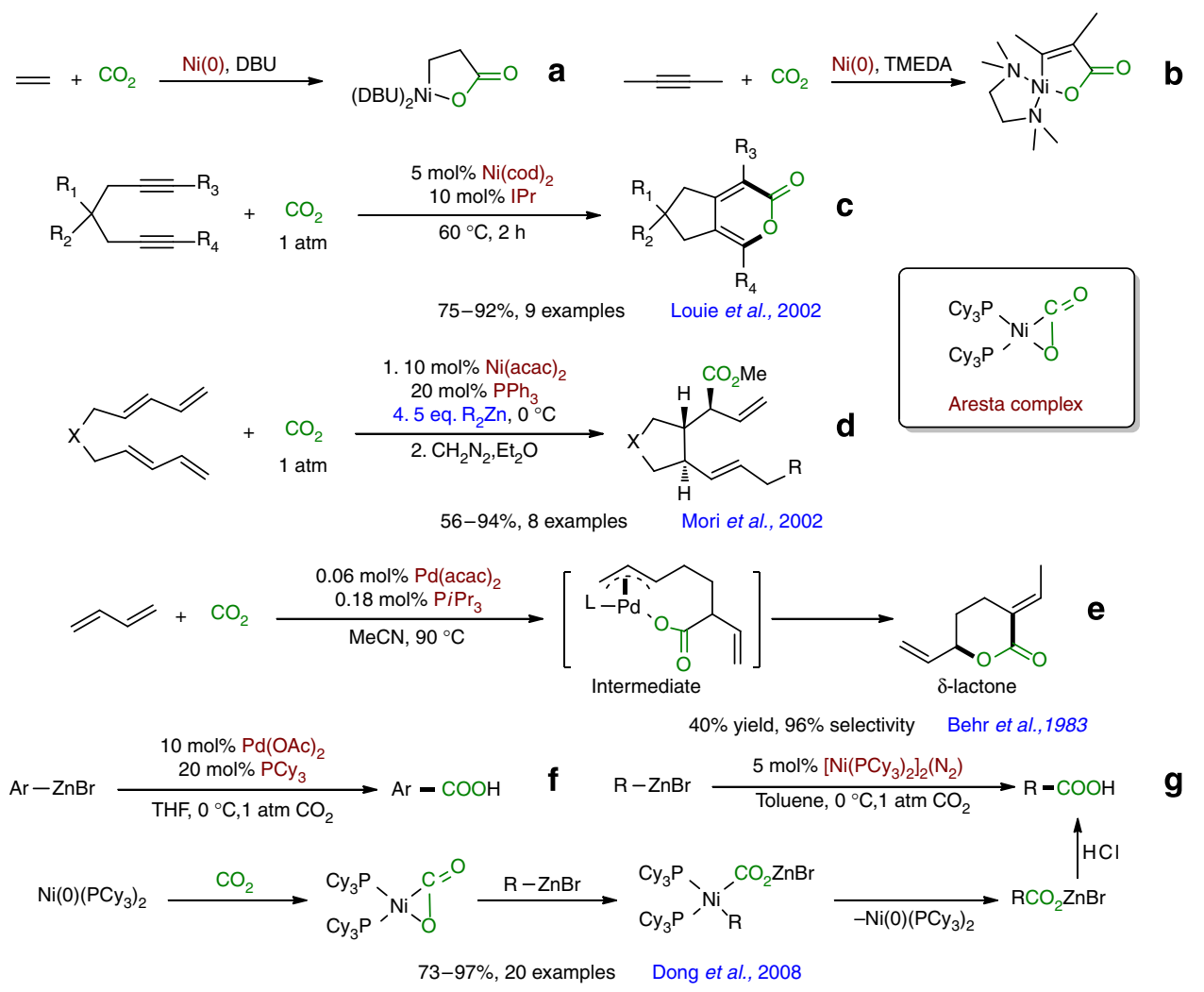

Figure 4 | Catalytic $\mathbf{C O}_{\mathbf{2}}$ fixation via oxidative cyclometallation with low-valent $\mathbf{N i}$ and $\mathbf{P d}$ complexes. (a,b) The generation of metallacycle complexes via the oxidative cyclometallation of $\mathrm{Ni}(0)$ complexes with $\mathrm{CO}_{2}$ and unsaturated bonds. (c) Ni-catalysed $[2+2+2]$ cycloaddition of $\mathrm{CO}_{2}$ and diynes. (e) Pd-catalysed telomerization of 1,3-butadiene with $\mathrm{CO}_{2}$. (f,g) Aresta's complex catalysed the carboxylation of organozinc reagents with $\mathrm{CO}_{2}$.

dioxide is an inert electrophile, highly reactive organolithium and Grignard reagents are typically utilized as strong nucleophiles to react with $\mathrm{CO}_{2}$ directly to construct $\mathrm{C}-\mathrm{C}$ bonds and furnish valuable carboxylic acids and their derivatives. In fact, the current industrial synthesis of salicylic acid derivatives is a prime example for this type of reaction. In general, nitrogen or oxygen nucleophiles exhibit much better reactivity than carbon nucleophiles for $\mathrm{CO}_{2}$ activation. As a result, catalytic $\mathrm{C}-\mathrm{C}$ bond formation using $\mathrm{CO}_{2}$ as $\mathrm{C} 1$ building block with less-reactive substrates has been and constitutes still a major challenging task for organic synthesis and catalysis research ${ }^{46}$. This goal can be achieved with the aid of transition metal catalysts that lower the activation energy and thus considerably increase the reaction rate for the $\mathrm{C}-\mathrm{C}$ bondforming step. Particularly, the $\mathrm{CO}_{2}$ insertion reactions into various $\mathrm{C}-\mathrm{M}$ bonds to form carboxylato complex via the formation of a new $\mathrm{C}-\mathrm{C}$ bond are well known ${ }^{47}$. Moreover, transition metal complexes can react with less-nucleophilic organometallic reagents, for example, organoboron compounds, as well as organohalides, unsaturated bonds even activated $\mathrm{C}-\mathrm{H}$ bonds to form C-M bonds efficiently, similar to their performance in the classic cross-coupling reactions ${ }^{48}$. Furthermore, the $\mathrm{CO}_{2}$ insertion intermediate (RCOO-M) might be quenched in a suitable way to regenerate the catalytically active species. This provides numerous possibilities for catalytic carboxylation reactions with all the above-mentioned substrates ${ }^{49-52}$. Representative examples for such processes are listed in Table 1 including a summary of substrates, catalyst precursors, ligands, additives as well as other conditions. In addition, a comparison of the postulated mechanisms for carboxylation and hydro(bora)carboxylation reactions are presented in Fig. 5.
Pioneered by the work of Iwasawa et al. in 2006 (Table 1, entry 1) the catalytic carboxylation of organoboron reagents with $\mathrm{CO}_{2}$ in the presence of $\mathrm{Rh}^{53}, \mathrm{Cu}^{54-57}$ and $\mathrm{Ag}$ (Table 1, entry 4) ${ }^{58}$ catalysts has been developed. These coupling reagents exhibit milder nucleophilicity, better stability to air and moisture as well as higher compatibility with sensitive functional groups. Among these results, the combination of IPrCu-NHC complex and alkoxide base $\left(\mathrm{M}^{\prime} \mathrm{OR}^{\prime}\right)$ developed by the group of Hou ${ }^{55,57}$ since 2008 was found to be the most active catalyst system for the carboxylation of aryl-, alkenyl- and also alkylboronic acid derivatives (Table 1, entries 3 and 6). A general synthesis of various carboxylic acids with $\mathrm{CO}_{2}$ was accomplished, and a wide range of functional groups were well tolerated. On the basis of stoichiometric experiments (taken the reactions of ArBPin, for example, as shown in Fig. 5a), a general mechanism for such reactions was also proposed: the metathesis reaction between $[(\mathrm{IPr}) \mathrm{CuCl}]$ and alkoxide generates the corresponding alkoxide complex $\left[(\mathrm{IPr}) \mathrm{Cu}\left(\mathrm{OR}^{\prime}\right)\right]$, which could coordinate with organoboron reagents $\left(\mathrm{R}-\mathrm{BX}_{2}\right)$ through interaction between the alkoxy group and the boron atom. Subsequent transmetalation to the copper atom generates the organocopper complex [(IPr)CuR]. Then, $\mathrm{CO}_{2}$ insertion into the $\mathrm{C}-\mathrm{Cu}$ bond yields the carboxylate [(IPr) $\mathrm{Cu}(\mathrm{OCOR})]$, which on metathesis with alkoxide base $\mathrm{M}^{\prime} \mathrm{OR}^{\prime}$ regenerates $\left[(\mathrm{IPr}) \mathrm{Cu}\left(\mathrm{OR}^{\prime}\right)\right]$ and releases the carboxylate salt $\mathrm{RCO}_{2} \mathrm{M}^{\prime}$. Finally, the latter yields the carboxylic acid $\mathrm{RCO}_{2} \mathrm{H}$ via hydrolysis. Some key reaction intermediates, such as the copper(I) alkoxide complexes [(IPr)Cu-(OR')], organocopper species [(IPr)CuR] and the carboxylate copper intermediates $[(\mathrm{IPr}) \mathrm{Cu}(\mathrm{OCOR})]$, were successfully isolated and structurally characterized, which were also confirmed to be catalytically active species in these transformations. 
Table 1 | A summary of carboxylation reactions of $\mathrm{C}-\mathrm{B}, \mathrm{C}-\mathrm{X}$ and $\mathrm{C}-\mathrm{H}$ bonds with $\mathrm{CO}_{2}$ : catalytic systems and reaction conditions.

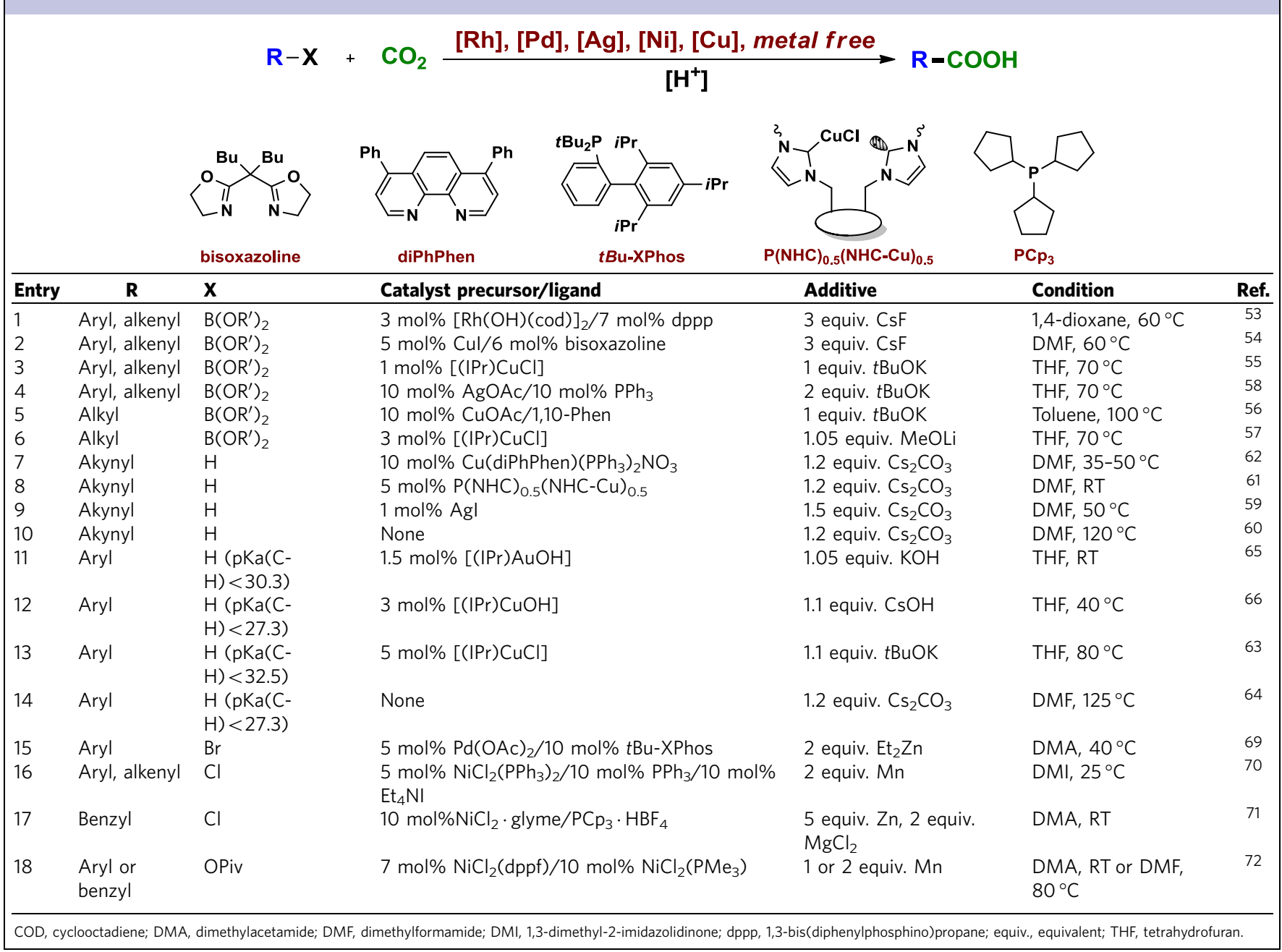

Apart from organoboron reagents, acidic $\mathrm{C}-\mathrm{H}$ bonds can also undergo deprotonative metallation with specific transition metal complexes to form $\mathrm{C}-\mathrm{M}$ bonds with the assistance of strong bases. Accordingly, the catalytic carboxylation of terminal alkynes (Table 1, entries 7-10) $)^{59-62}$ and electron-deficient (hetero)arenes (Table 1 , entries $11-14)^{63-66}(\mathrm{pKa}(\mathrm{C}-\mathrm{H})<32.5)$ is achieved through the following $\mathrm{CO}_{2}$ insertion and metathesis of metal carboxylate intermediates with base. Except the active copper and gold $N$-heterocyclic carbene complexes, $\mathrm{Cu}($ diPhPhen $)\left(\mathrm{PPh}_{3}\right)_{2} \mathrm{NO}_{3}$ (Table 1, entry 7) and AgI (Table 1, entry 9) were also demonstrated to be the effectual catalysts for the carboxylation of terminal alkynes by the group of Goossen ${ }^{62}$ and $\mathrm{Lu}^{59}$, respectively. Interestingly, at higher temperature $\left(>120^{\circ} \mathrm{C}\right.$ ), the $\mathrm{Cs}_{2} \mathrm{CO}_{3}$-mediated carboxylation of heteroarenes (Table 1 , entry 14$)^{64}$ and terminal alkynes (Table 1 , entry 10$)^{60}$ with $\mathrm{CO}_{2}$ were reported by $\mathrm{Hu}$ and Zhang's groups independently as well.

Later on, the group of Iwasawa showed the formation of C-M bonds by a directing group-assisted $\mathrm{C}-\mathrm{H}$ activation process, which was used for subsequent carboxylation reactions with $\mathrm{CO}_{2}$. In fact, treatment of 2-hydroxystyrenes bearing a hydroxy group as the chelating group with catalytic amount of $\mathrm{Pd}(\mathrm{OAc})_{2}$ in the presence of $\mathrm{Cs}_{2} \mathrm{CO}_{3}$ and $\mathrm{CO}_{2}$ afforded the corresponding coumarins via carboxylation/condensation (Fig. $5 \mathrm{c}$ ) ${ }^{67}$. It is proposed that first the six-membered alkenyl palladium intermediate is generated by chelation-assisted alkenyl C-H bond cleavage of 2-hydroxystyrene with $\mathrm{Pd}(\mathrm{OAc})_{2}$ along with coordination of the second molecule of substrate. Subsequently, the alkenyl palladium(II) species undergoes nucleophilic carboxylation to afford a palladium carboxylate intermediate, which reacts with 2-hydroxystyrene and base to give lactone with regeneration of the cyclometalated intermediate. The same group also reported another approach for the direct carboxylation reaction of aromatic $\mathrm{C}-\mathrm{H}$ bonds: namely, the $\mathrm{Rh}(\mathrm{I})$-catalysed carboxylation of aromatic compounds via chelation-assisted ortho-metalation (Fig. 5d) ${ }^{68}$. The key intermediate is proposed to be the methylrhodium(I) complex $\mathbf{F}$, which undergoes chelation-assisted oxidative addition with a suitable C-H bond to afford rhodium(III) complex G, followed by reductive elimination of methane to give the nucleophilic arylrhodium(I) complex $\mathbf{H}$. Consequently, nucleophilic carboxylation of intermediate $\mathbf{H}$ affords rhodium carboxylate $\mathbf{I}$, which undergoes transmetalation with a methylaluminium reagent to give aluminium carboxylate $\mathbf{J}$ and regenerate the active methylrhodium(I) complex $\mathbf{F}$.

The oxidative addition of organo(pseudo)halides with lowvalent transition metals constitutes another way to form C-M bonds. In this respect, in 2009, Martin and co-workers ${ }^{69}$ presented a palladium-catalysed direct carboxylation of aryl bromides with $\mathrm{CO}_{2}$ (Table 1, entry 15). In contrast to the reactions vide supra, this is the first example for carboxylation reactions of electrophiles instead of nucleophiles. Therefore, additional reductant (here $\mathrm{Et}_{2} \mathrm{Zn}$ ) is needed to regenerate the 
a
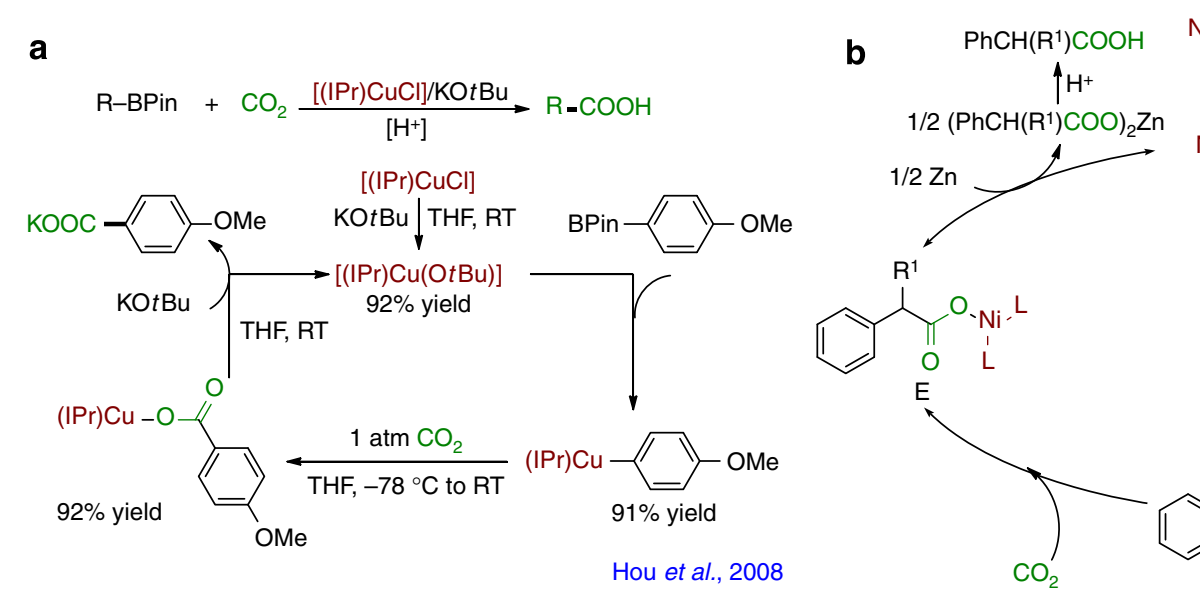

$\mathrm{O}$
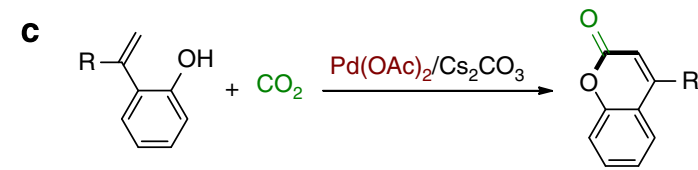

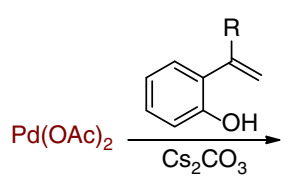
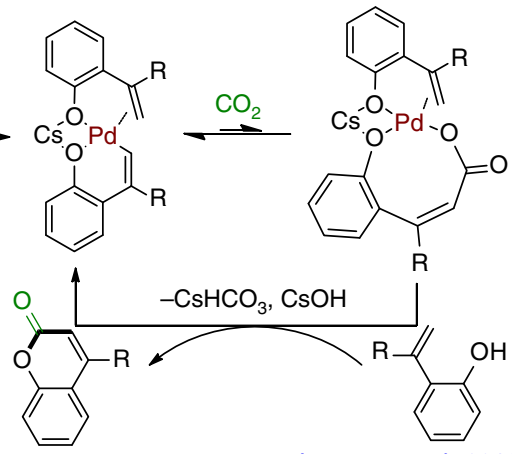

$\mathrm{R}$

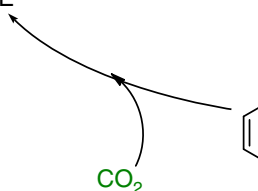

$\mathrm{CO}_{2}$

Martin et al., 2013

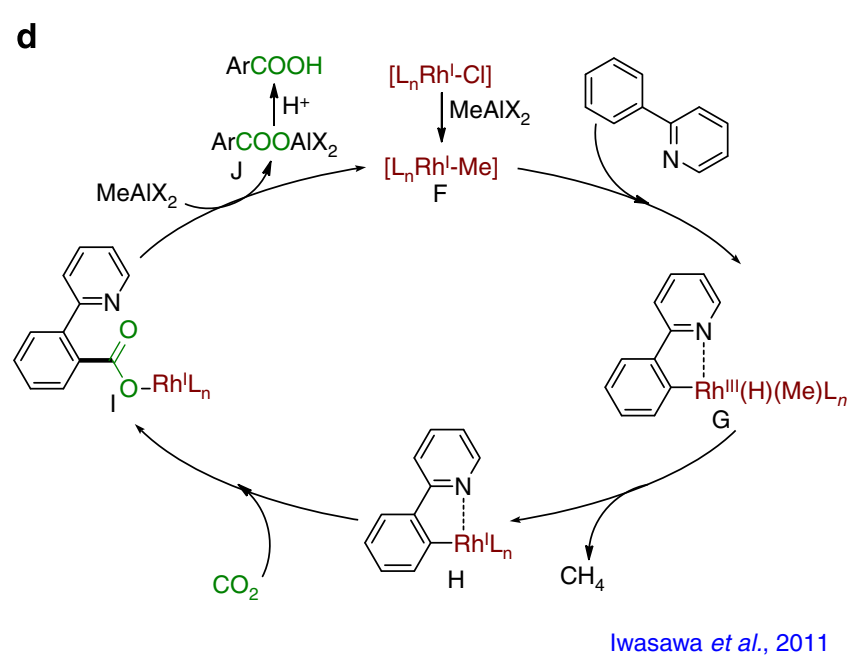

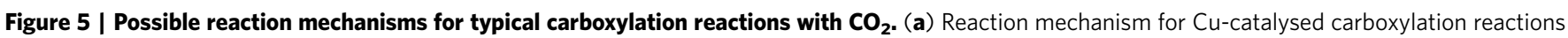
of organoboron compounds. (b) Reaction mechanism for Ni-catalysed carboxylation reactions of benzyl halides. (c,d) Directing group-assisted carboxylation of $\mathrm{C}-\mathrm{H}$ bonds.

catalytically active low-valent $\mathrm{Pd}(0)$ species. After Martin's work, Tsuji and co-workers found that less-expensive nickel catalysts are active in carboxylation of aryl chlorides and vinyl chlorides. Notably, these reactions proceed under $1 \mathrm{~atm} \mathrm{CO}_{2}$ pressure at room temperature (Table 1 , entry 16$)^{70}$. The $\mathrm{Ni}(\mathrm{I})$ species was involved in the catalytic cycle with the aid of the $\mathrm{Mn} / \mathrm{Et}_{4} \mathrm{NI}$ system as an efficient reducing agent. Although the carboxylation of aryl bromides and chlorides with $\mathrm{CO}_{2}$ provides a straightforward synthetic route to functionalized benzoic acids, these protocols cannot be utilized to access aliphatic electrophiles, for example, for the synthesis of biologically active phenylacetic acid derivatives. Hence, it was interesting when Martin's group reported in 2013 the first catalytic carboxylation of primary, secondary and tertiary benzyl halides utilizing $\mathrm{CO}_{2}$. Their protocol provides an opportunity to prepare phenylacetic acids from commercially available and cheap starting materials (Table 1 , entry 17$)^{71}$. The proposed catalytic cycle starts with an initial reduction of the $\mathrm{Ni}(\mathrm{II})$ pre-catalyst by $\mathrm{Zn}$ followed by oxidative addition to the corresponding alkyl halides, thus delivering $\eta^{3}$ - and $\eta^{1}$-bound nickel complexes $\mathbf{B}$ and $\mathbf{C}$ in a rapid equilibrium. Generation of the reduced $\mathrm{Ni}(\mathrm{I})$ species $\mathbf{D}$ by $\mathrm{Zn}$ and $\mathrm{CO}_{2}$ insertion affords the carboxylate nickel intermediate E. Further treatment with $\mathrm{Zn}$ regenerates the active $\mathrm{Ni}(0) \mathrm{L}_{2}$ A species and provides zinc carboxylate, which on hydrolysis delivers the final product (Fig. 5b). Very recently, the same group reported a novel $\mathrm{Ni}$-catalysed carboxylation of aryl esters with less-activated C-O bonds (Table 1 , entry 18) ${ }^{72}$. This new protocol deals with the development of a synergistic activation of $\mathrm{CO}_{2}$ and a rather challenging activation of inert $\mathrm{C}\left(\mathrm{sp}^{2}\right)-\mathrm{O}$ and $\mathrm{C}\left(\mathrm{sp}^{3}\right)-\mathrm{O}$ bonds derived from simple and cheap alcohols. In general, all these modern transformations have to be compared with the classic carboxylation of Grignard reagents. The main advantage of these recent methodologies can be the tolerance of functional groups, and thus the possibility to synthesize complex carboxylic acid derivatives. On the other hand, the major drawback of all carboxylation reactions of electrophiles is the necessity to use (over)stoichiometric amounts of metals as reducing agents. Obviously, hydrogen would be a much more favourable reductant for such processes; however, no efficient regeneration of the active low-valent metal complexes have been realized yet.

Significant advancements were made in the catalytic $\mathrm{CO}_{2}$ hydrocarboxylation of unsaturated $\mathrm{C}-\mathrm{C}$ bonds too. For example, in 2008, Iwasawa and co-workers reported the hydrocarboxylation of allenes and 1,3-dienes with a silyl pincer-type palladium complex. The reactions were carried out at room temperature under $1 \mathrm{~atm} \mathrm{CO}_{2}$ and enabled the facile and regioselective synthesis of $\beta, \gamma$-unsaturated carboxylic acids ${ }^{73}$. The reaction is believed to start with generation of a silyl pincer-type palladium hydride complex via transmetalation of $\mathrm{AlEt}_{3}$ to (PSiP)PdOTf 
followed by $\beta$-hydride elimination. Hydrometalation of 1,1disubstituted allenes preferentially afford the less-congested $\sigma$-allylpalladium intermediate, which then undergoes nucleophilic addition to $\mathrm{CO}_{2}$ at the $\gamma$-position of the palladium regioselectively to give the carboxylate palladium complex. Finally, a transmetalation/ $\beta$-hydride elimination sequence regenerates the catalytically active palladium hydride species and releases the carboxylation product as its $\mathrm{Al}$ salt (Fig. 6a). Meanwhile, the first direct hydrocarboxylation of alkenes occurred in similar manner. Rovis and co-workers ${ }^{74}$ developed a Ni-catalysed hydrocarboxylation of styrenes using $\mathrm{Et}_{2} \mathrm{Zn}$ as the hydride donor with $\mathrm{CO}_{2}$ (Fig. 6b). The suggested mechanism involved a nickel hydride complex as active catalyst. Afterwards, a highly regio- and stereoselective three-component $\mathrm{Ni}$-catalysed syn-hydrocarboxylation of alkynes with $\mathrm{Et}_{2} \mathrm{Zn}$ and $\mathrm{CO}_{2}$ using $\mathrm{Ni}(\operatorname{cod})_{2}$ as catalyst and CsF as additive was presented by Ma and co-workers ${ }^{75}$. Although these three examples demonstrate interesting carboxylation reactions, strong and extremely airsensitive reducing agents are indispensable. This problem is partially avoided in the copper-catalysed hydrocarboxylation of alkynes with $\mathrm{CO}_{2}$ using hydrosilanes as milder reducing agent realized by Tsuji and co-workers (Fig. $6 c^{76}$. The fundamental catalytic steps in their hydrocarboxylation were examined by stoichiometric reactions. Treatment of the catalyst precursor $\left[\mathrm{Cl}_{2} \mathrm{IPrCuF}\right]$ with silane led to the catalytically active copper

a

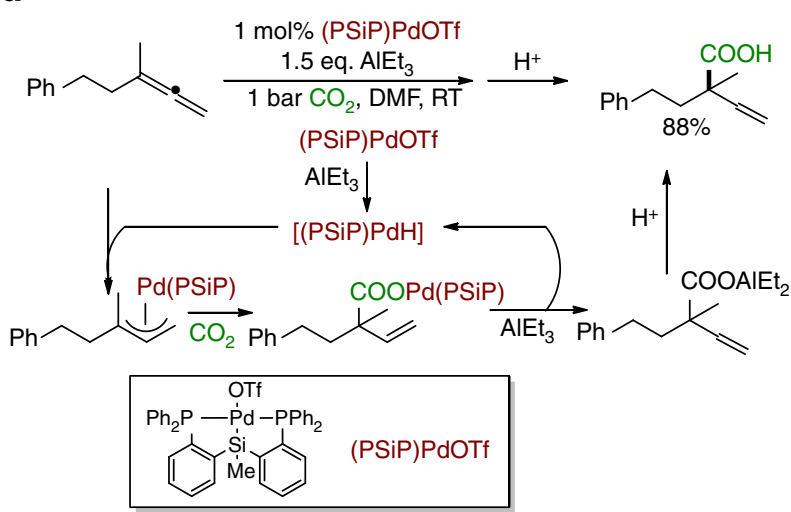

Iwasawa et al., 2008

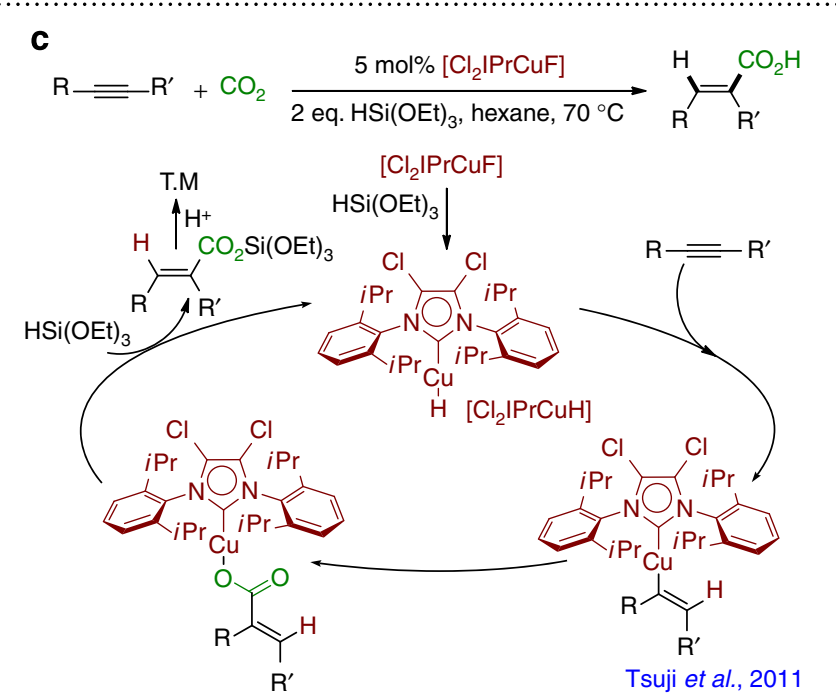

hydride species. It reacted with an alkyne substrate smoothly to afford the corresponding copper alkenyl complex, which on $\mathrm{CO}_{2}$ insertion affords the copper carboxylato complex. $\delta$-bond metathesis of this species with hydrosilane regenerated $\left[\mathrm{Cl}_{2} \mathrm{IPrCuH}\right]$ and provided the corresponding silyl ester. In analogy to hydrocarboxylations, catalytic boracarboxylations of alkynes with diborane and $\mathrm{CO}_{2}$ using an $\mathrm{N}$-heterocyclic carbene copper catalyst were recognized by Hou in 2012 (ref. 77). The reaction of $[(\mathrm{NHC}) \mathrm{Cu}(\mathrm{OR})]$ with $\mathrm{B}_{2}(\text { pin })_{2}$ instantly gave the borylcopper complex $[(\mathrm{NHC}) \mathrm{CuB}($ pin) $)$. Its subsequent reaction with internal alkyne afforded a $\beta$-boryl alkenyl complex, which undergoes the following $\mathrm{CO}_{2}$ insertion and metathesis processes to furnish the final product (Fig. 6d).

Carbonylation reactions using $\mathrm{CO}_{2}$ as $\mathrm{CO}$ surrogates. Compared with all above strategies to directly use $\mathrm{CO}_{2}$ as $\mathrm{C} 1$ building block in chemical synthesis, the in situ generation of $\mathrm{CO}$ from $\mathrm{CO}_{2}$ is clearly underdeveloped. Nevertheless, this strategy represents a particularly promising tool for more practical applications. We are convinced that such $\mathrm{CO}_{2}$ valorization approach will open up new opportunities to realize industrially important carbonylation reactions. Advantageously, here $\mathrm{CO}_{2}$ is used as a sustainable carbonyl source instead of hazardous $\mathrm{CO}$. For more than 60 years, carbonylation reactions using $\mathrm{CO}$

b
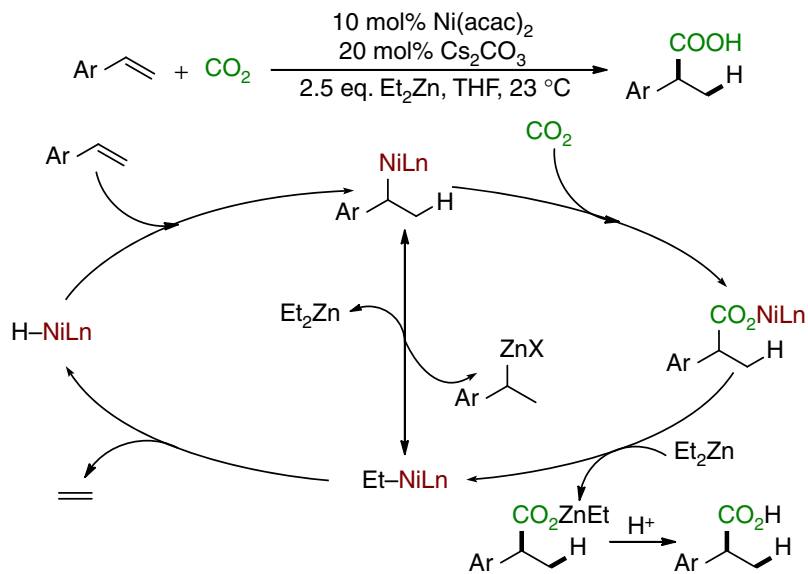

$\mathrm{R}=\mathrm{R}^{\prime}+\mathrm{B}_{2}(\mathrm{pin})_{2}+\mathrm{CO}_{2} \frac{5 \mathrm{~mol} \%[(\mathrm{SIMes}) \mathrm{CuCl}]}{\begin{array}{l}1.1 \text { eq. LiOtBu} \\ \mathrm{THF}, 80^{\circ} \mathrm{C}, 14 \mathrm{~h}\end{array}} 又_{\mathrm{R}}^{\mathrm{O}}$
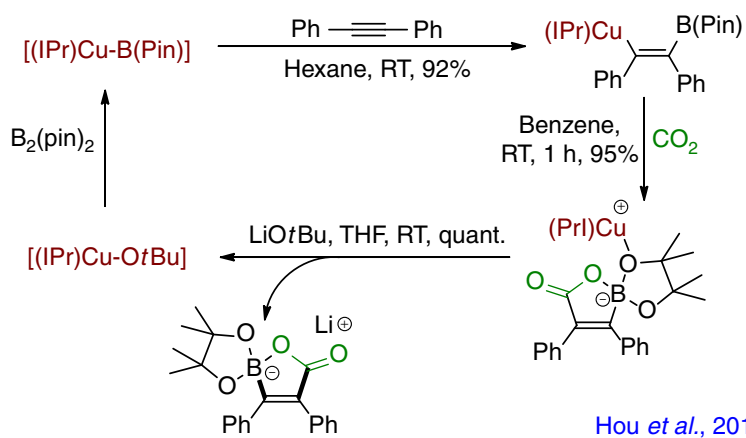

Hou et al., 2012

Figure 6 | Selected examples for hydro(bora)carboxylation reactions of alkenes and alkynes with $\mathrm{CO}_{2}$. Examples include (a) allenes, (b) styrenes and (c,d) internal alkynes. 
represent major technology for the production of value-added bulk and fine chemicals from easily available feedstocks. As an example, over 10 million tons of oxo-products are produced every year from alkenes via hydroformylation and related tandem reactions ${ }^{78}$. Besides, methyl propionate, a key intermediate for methyl methacrylate (annual demand is currently about 3 million tons), is in part produced by palladium-catalysed methoxycarbonylation of ethylene ${ }^{79}$. So far, all these processes make use of CO, which is also difficult to transport on a bulk scale. Notably, the physical properties of carbon monoxide (especially its toxicity) impede its wider use in academia too. In this respect, carbonylations with $\mathrm{CO}_{2}$ offer a more safe and accessible tool for synthetic organic chemists. The general strategy to use carbon dioxide as a carbonyl source is based on the in situ reduction of $\mathrm{CO}_{2}$ to $\mathrm{CO}$, which then can be used in subsequent carbonylation reactions. It is long known that combination of carbon dioxide and $\mathrm{H}_{2}$ at high temperature $\left(>300^{\circ} \mathrm{C}\right.$ ) allows for so-called reverse water gas shift (RWGS) reaction, in which a mixture of carbon monoxide, carbon dioxide, hydrogen and water is formed. On the basis of their previous studies on the RWGS process, Tominaga and Sasaki et al. ${ }^{80}$ reported in 2000 the first ruthenium-catalysed hydroformylation/ reduction of alkenes with $\mathrm{CO}_{2}$ using $\mathrm{Ru}_{3}(\mathrm{CO})_{12}$ as catalyst in the presence of $\mathrm{LiCl}$ as additive. It is surprising that this seminal work of Tominaga et al. has attracted only minor attention, probably due to the still drastic conditions and the efficiency of the reaction. For example, unwanted alkene hydrogenation side reactions occurred. Recently, our group improved this process by the application of a specific bulky phosphite ligand (Fig. 7a) ${ }^{81}$. Applying the optimized catalytic system, the yields of alcohols, the chemoselectivity of the process and especially the TON of the catalyst was significantly improved compared with the known ligand-free catalysis system. In the above-mentioned carbonylation reactions via RWSG reaction, water is always produced as a side product and is not involved in the following alkene carbonylation reactions. Remarkably, the group of Leitner $^{82}$ reported a rhodium-catalysed hydrocarboxylation of alkenes with $\mathrm{CO}_{2}$ and $\mathrm{H}_{2}$ to produce carboxylic acids, in which in situ-formed $\mathrm{H}_{2} \mathrm{O}$ was utilized as the nucleophile in this transformation (Fig. 7b).

Although $\mathrm{H}_{2}$ can act as an efficient reducing reagent for $\mathrm{CO}_{2}$ reduction, in many cases the following carbonylation reaction does not tolerate $\mathrm{H}_{2}$ due to the hydrogenation of the substrate. As a result, the development of other benign $\mathrm{CO}_{2}$ reductions to generate $\mathrm{CO}$ is highly desired for the valorization of $\mathrm{CO}_{2}$. Combining our long-term experience in alcohol dehydrogenation $^{83}$ and carbon dioxide reduction reactions $s^{5,84}$, very recently efficient reforming processes of alcohols and $\mathrm{CO}_{2}$ were developed. This allows for catalytic alkoxycarbonylation reactions using $\mathrm{CO}_{2}$ as the $\mathrm{C} 1$ source in the presence of a ruthenium/[Bmim $] \mathrm{Cl}$ catalyst system. Key to success was an unprecedented in situ formation of $\mathrm{CO}$ from $\mathrm{CO}_{2}$ (Fig. $\left.7 \mathrm{c}\right)^{85}$. In contrast to previously reported hydrocarboxylation reactions of alkenes with $\mathrm{CO}_{2}$, similar carboxylated products are obtained, but the presence of stoichiometric amounts of strong and expensive reductants like $\mathrm{Et}_{2} \mathrm{Zn}$ and silanes is avoided. The following reaction steps are proposed: initially, $\mathrm{CO}_{2}$ is reduced to $\mathrm{CO}$ (path I) or alkyl formates (path II) through transfer hydrogenation with alcohols. Path I is proposed to be the major reaction pathway for this transformation based on the control experiments. Meanwhile, the alcohol undergoes dehydrogenation to form the corresponding carbonyl compound (path III). Interestingly, a minor amount of the $\mathrm{CO}$ comes from further decarbonylation reactions of the alcohol as shown by isotope-labelling experiments. It is estimated that this novel methodology is applicable to other carbonylation reactions as well and will contribute substantially to the development of next-generation $\mathrm{CO}_{2}$ utilization. Apart from $\mathrm{CO}_{2}$ reduction with hydrogen and alcohols, Skrydstrup and coworkers shortly afterwards reported the successful identification of an efficient conversion of $\mathrm{CO}_{2}$ to $\mathrm{CO}$ performed at room temperature with catalytic caesium fluoride in the presence of a disilane, which can be coupled to Pd-catalysed amino- and alkoxycarbonylations with aryl bromides and iodides ${ }^{86}$.

Inspired by nature's efficiency in converting carbon dioxide into carbohydrates and other organic matter through photosynthesis, chemists have also explored opportunities to directly reduce $\mathrm{CO}_{2}$ to $\mathrm{CO}$ by photocatalytic means. Since $\mathrm{CO}_{2}$ does not absorb either visible or ultraviolet radiation in the wavelengths of $200-700 \mathrm{~nm}$, this process requires a suitable photocatalyst to absorb ultraviolet-visible radiation and transfer it to $\mathrm{CO}_{2}$. Photosensitizers (PSs) that meet this criterion often include a metal centre, as the $d$-orbital splitting in organometallic species often falls within the energy range of far-ultraviolet and visible light. The reduction process begins with excitation of the PS. This causes the transfer of an electron from the metal centre into the coordinated ligands. This movement is termed as metal-to-ligand charge transfer process. Back-electron transfer from the ligands to the metal after the charge transfer is prevented by including an electron-donating species to quench the exciting state of the photocatalyst, thus forming the one-electron-reduced (OER) intermediate for further $\mathrm{CO}_{2}$ reduction processes ${ }^{87}$.

Initial work by Lehn and co-workers in the 1980s led the development of first photocatalytic $\mathrm{CO}_{2}$ reductions. Here, $\mathrm{CoCl}_{2}$ was used as the catalytic site for $\mathrm{CO}_{2}$ reduction with $\left[\mathrm{Ru}(\text { bpy })_{3}\right] \mathrm{Cl}_{2}$ as PS (Fig. $\left.7 \mathrm{~d}\right)^{88}$. However, $\mathrm{CoCl}_{2}$ has very low selectivity towards the reduction of $\mathrm{CO}_{2}$ to $\mathrm{CO}$, resulting from the reduction of water in the aqueous media to produce $\mathrm{H}_{2}$ that is the main product in this system. The TON of $\left(\mathrm{CO}+\mathrm{H}_{2}\right)$ is only 32 based on $\left[\mathrm{Ru}(\mathrm{bpy})_{3}\right] \mathrm{Cl}_{2}$. The efficiency of these photocatalytic processes is also characterized by its quantum yield $\Phi$, which is defined as the number of times a $\mathrm{CO}_{2}$ molecule is transformed per photon absorbed by the PS. However, this system reached a quantum yield of only $1.2 \%$. Despite the low selectivity and efficiency of this reaction, the simultaneous reduction of $\mathrm{CO}_{2}$ and $\mathrm{H}_{2} \mathrm{O}$ produces in fact syngas, which is of interest for large-scale industrial processes. Moreover, it represents an early step in the development of chemical systems capable of artificial photosynthesis and solar energy conversion. Later, the same research group found that rhenium-based complexes $\left[\operatorname{Re}(\right.$ bipy $\left.)(\mathrm{CO})_{3} \mathrm{X}\right](\mathrm{X}=\mathrm{Cl}, \mathrm{Br})$ acting as both $\mathrm{PS}$ and catalyst are more efficient and represent more selective catalysts for $\mathrm{CO}_{2}$ reduction ${ }^{89}$. The process has very high $\mathrm{CO} / \mathrm{H}_{2}$, selectivity, generating only $\mathrm{CO}$, and affords a quantum yield of up to $14 \%$. It is indicated that the dissociation of the ligand $\mathrm{X}^{-}$from the unstable 19-electron OER is a key step in this photocatalytic reduction of $\mathrm{CO}_{2}$ (ref. 90). In addition, Fujita and co-workers identified the binuclear [ $\mathrm{Re}-\mathrm{C}(\mathrm{O}) \mathrm{O}-\mathrm{Re}]$ moiety as a key intermediate of the two-electron reduction process from $\mathrm{CO}_{2}$ to CO (ref. 91). In 2008, based on their insightful mechanistic study, the most efficient photocatalytic system was successfully developed by the group of Ishitani using a mixed catalysts' system with $\mathrm{fac}$ - $\left[\mathrm{Re}(\mathrm{bpy})(\mathrm{CO})_{3}\left(\mathrm{CH}_{3} \mathrm{CN}\right)\right]^{+}$and $\mathrm{fac}$-[Re-4,4'$(\mathrm{MeO})_{2}$ bpy $\left.(\mathrm{CO})_{3} \mathrm{P}(\mathrm{OEt})_{3}\right]^{+}$affording the optimized quantum yield for CO formation was 0.59 (ref. 92).

Despite the improved activity of rhenium polypyridine complexes for $\mathrm{CO}_{2}$ reduction, a major problem with these photocatalysts is the lack of an extended absorption into the visible region. To solve this problem, Ishitani and Bian and coworkers presented the use of covalently linked heteronuclear $\mathrm{Ru}$ and Re multimetallic complexes in the photocatalytic reduction of $\mathrm{CO}_{2}$ (refs 93,94). Enhancement of the photocatalytic response to light in the visible region was achieved by specifically designed 

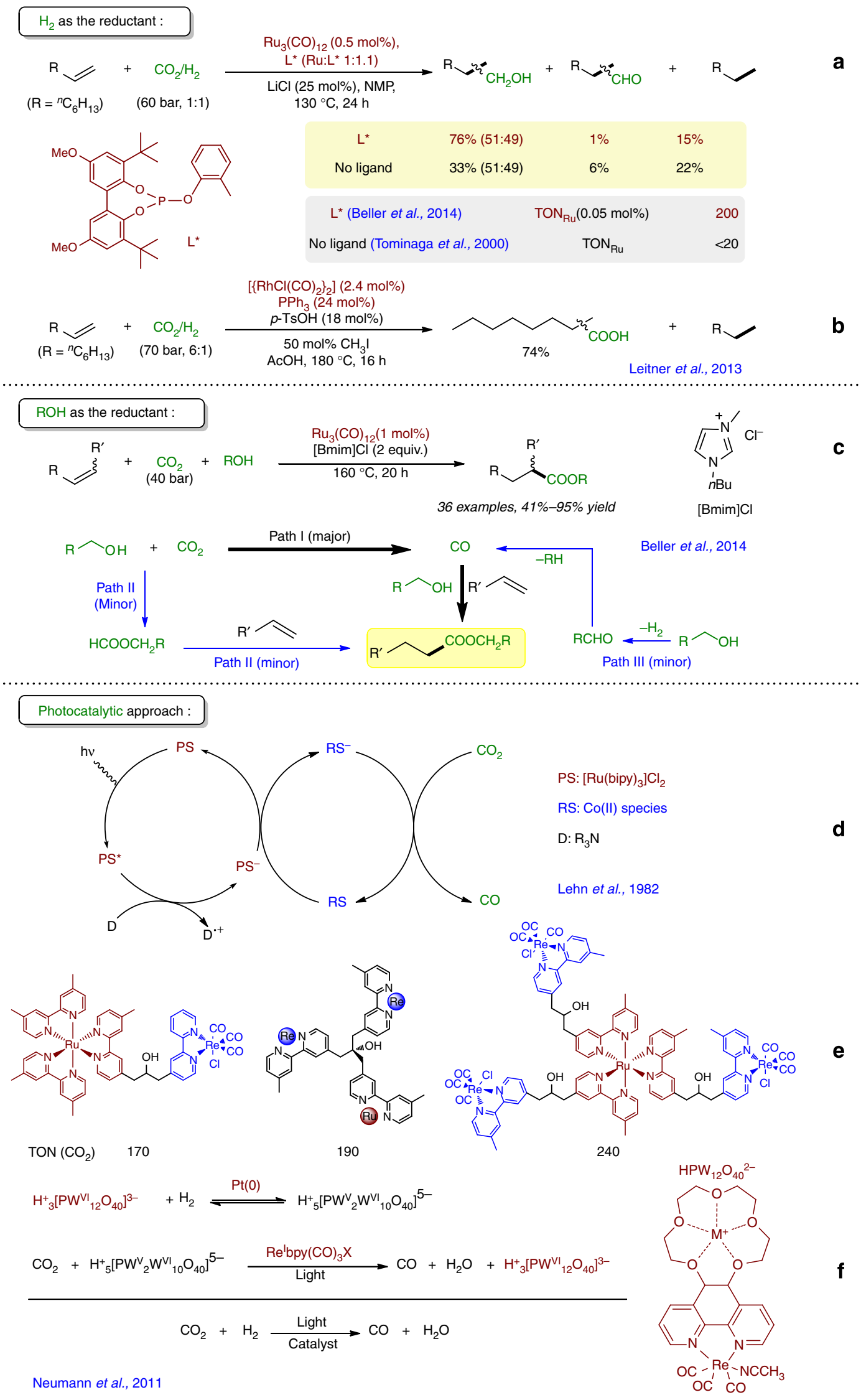

Figure 7 | Catalytic production of $\mathbf{C O}$ from $\mathbf{C O}_{\mathbf{2}}$ reduction and its application in carbonylation reactions. (a,b) $\mathrm{H}_{2}$ as the reductant. (c) Alcohol as the reductant. (d-f) Photocatalytic approach. 
supramolecular complexes, and their high photocatalytic activities were attributed to intramolecular electron transfer from the OER $\mathrm{Ru}$ species to the $\mathrm{Re}$ moiety. The binuclear complex $\mathrm{Ru}-\mathrm{Re}$, trinuclear complex $\mathrm{RuRe}_{2}$ and tetranuclear complex $\mathrm{RuRe}_{3}$ all furnished higher TON of CO (Fig. 7e). Despite recent improvements ${ }^{95}$, it should be noted that, in the above-mentioned photochemical $\mathrm{CO}_{2}$ reduction, common sacrificial donors such as triethylamine (TEA), triethanolamine (TEOA) and 1-benzyl1,4-dihydronicotinamide (BNAH) have to be used. From a practical point of view, it is noteworthy that in 2011 Neumann and co-workers ${ }^{96}$ developed a photoreduction of $\mathrm{CO}_{2}$ to $\mathrm{CO}$ with $\mathrm{H}_{2}$ catalysed by a rhenium(I) phenanthroline-polyoxometalate hybrid complex. In this study, previously used sacrificial electron donors were replaced by $\mathrm{H}_{2}$. The working catalysis system is summarized in Fig. 7f. On one hand, a polyoxometalate oxidizes $\mathrm{H}_{2}$ to two protons and two electrons in the presence of $\mathrm{Pt}(0)$. On the other hand, the reduced form of the polyoxometalate reacts with $\mathrm{CO}_{2}$ with the assistance of a chromophoric rhenium(I) complex and visible light to produce $\mathrm{CO}$ and $\mathrm{H}_{2} \mathrm{O}$. As a result, the overall reaction is a photocatalytic RWGS reaction, which is of interest for both industry and academia for its further potential application in carbonylation reaction with $\mathrm{CO}_{2}$.

\section{Future perspectives}

Today's chemical production as well as academic organic synthesis relies mainly on the use of fossil-based carbon sources. In contrast, the increased valorization of $\mathrm{CO}_{2}$ as a recyclable and abundant $\mathrm{C} 1$ building block would allow for the development of a sustainable chemical industry. In this respect, the development of benign transformations of carbon dioxide is a highly attractive object for synthetic chemistry. Clearly, in the past decades, several effective activation modes for catalytic $\mathrm{CO}_{2}$ fixation and a series of novel transformations have been disclosed.

Issues need to be resolved. Despite the impressive progress, the efficiency of most reactions is far from satisfying. Hence, only recent new processes utilizing $\mathrm{CO}_{2}$ as the $\mathrm{C} 1$ source have been industrialized. In most methodology-driven work, there remain important issues that need to be addressed in the future, for example, less utilization of very reactive/sensitive reagents, extending substrate scope, milder reaction conditions, improved chemo- and regioselectivity. Due its inherent inertness, not surprisingly, reactions of carbon dioxide under mild conditions need a thermodynamic driving force, for example, energy-rich coupling partners. The copolymerization of epoxides with $\mathrm{CO}_{2}$ to produce polycarbonates is an elegant example for this strategy and permits efficient $\mathrm{CO}_{2}$ valorization. Alternative copolymerizations with easily available chemical feedstocks, such as olefins, are highly desired too. In this respect, the recent report from Nozaki's group of a copolymerization of $\mathrm{CO}_{2}$ and 1,3-butadiene is interesting. This work provides a straightforward approach towards the production of novel polymeric materials from $\mathrm{CO}_{2}$ (ref. 97).

Another promising field might be the use of carbon dioxide as methylation agent. For example, ruthenium-catalysed methylation of amines with $\mathrm{CO}_{2}$ and $\mathrm{H}_{2}$ to afford valuable products has very recently been achieved. However, the current catalyst loading is still too high for large-scale production. Hence, the development of more efficient catalyst systems and non-noble metal-based catalysts for this and related transformations constitutes a demanding goal.

Most of the $\mathrm{CO}_{2}$-fixation reactions, which proceed via oxidative cyclometallation pathway, are restricted to extensive $\pi$ systems (dienes and diynes) as substrates and the use of zinc reagents to cleave the metallacycle intermediates. Consequently, utilization of other simple alkene or alkyne substrates and milder nucleophiles is highly desired to further improve this strategy.
Carboxylation reactions via $\mathrm{CO}_{2}$ insertion into $\mathrm{C}-\mathrm{M}$ bonds in combination with classical coupling reactions are intensively studied in the past 10 years. Although this strategy furnished a series of interesting transformations, the requirement of stoichiometric amounts of strong bases, sensitive metal reagents or expensive hydrosilanes are evident drawbacks that need to be overcome. The key point to solve such problems in the future will be the invention of new routes to quench metal carboxylate complex (RCOOM) with less-nucleophilic agents to afford the final product and regenerate the original catalytically active species.

Finally, it is worth noting that the catalytic in situ generation of $\mathrm{CO}$ from $\mathrm{CO}_{2}$ and its recent applications in sequential carbonylation reactions is a more favourable strategy for $\mathrm{CO}_{2}$ valorization. It provides the use of safe and abundant $\mathrm{CO}_{2}$ instead of $\mathrm{CO}$ as carbonyl source. Here the improvement of (regio)selectivities and to use milder reaction conditions represent important targets. So far, most catalysts in such reactions are ruthenium-based complexes. Encouraged by the recently developed highly regioselective ruthenium-catalysed hydroformylation reaction ${ }^{98,99}$, in the future more efforts should be donated to solve the regioselectivity problem with suitable ligands and/or additives. Last but not the least, similar to other catalysis processes, the discovery of nonnoble metal-catalysed reactions, for example, iron catalysts, is an essential issue in this field. Although related photocatalytic approaches proceed under very mild conditions, the TON is too low currently. Probably, cascade reaction sequences that make use of carbonylation processes to consume the in situ-generated $\mathrm{CO}$ will drive the overall reaction more efficiently.

Towards artificial photosynthesis. Photosynthesis basically supplies the carbon source for all organic compounds on Earth. It is an ideal green process for the conversion of light energy into chemical energy, which makes use of $\mathrm{CO}_{2}$ (Fig. 8, equation a). Inspired by the nature's strategy, the development of sustainable and straightforward catalytic C-C bond-forming reactions of $\mathrm{CO}_{2}$ in industrially relevant target molecules will be the 'crown jewel' of organic synthesis and catalysis research in the future. We expect several dream reactions towards artificial photosynthesis $\left(\mathrm{CO}_{2}\right.$ reduction under very mild conditions) and beyond will become true in the coming years. For example, sodium acrylate is an industrially important acrylate, with a global market volume of about 4 million tons and is commonly used as a monomer in the production of superabsorber polymers. Thus, the direct synthesis of acrylic acid from $\mathrm{CO}_{2}$ and ethylene represents an economically attractive but also most challenging dream reaction. Until now, a TON of only 10 ( $\mathrm{Ni}$ catalyst and $\mathrm{NaOt} \mathrm{Bu}$ as base) has been realized; nevertheless, the possibility of this reaction was clearly demonstrated (Fig. 8, equation b) ${ }^{100}$. Ethylene glycol is another important raw material in the manufacture of polyester fibres and polyethylene terephthalate resins used in bottling. The direct

$$
\begin{aligned}
& 6 \mathrm{CO}_{2}+6 \mathrm{H}_{2} \mathrm{O} \underset{\text { hv }}{\stackrel{\text { Cat. }}{\longrightarrow}} \mathrm{C}_{6} \mathrm{H}_{12} \mathrm{O}_{6}+6 \mathrm{O}_{2} \\
& \mathrm{CO}_{2}+=\stackrel{\text { Cat. }}{\longrightarrow} \sim \mathrm{CO}_{2} \mathrm{H}
\end{aligned}
$$

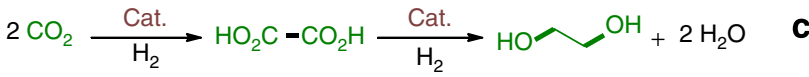

$$
\begin{aligned}
& \mathrm{CO}_{2}+3 \mathrm{H}_{2}+\square \stackrel{\text { Cat. }}{\longrightarrow} \longrightarrow \mathrm{CH}_{3}+2 \mathrm{H}_{2} \mathrm{O}
\end{aligned}
$$

Figure 8 | Dream reactions towards artificial photosynthesis. (a)

Photosynthesis reaction process. (b) Direct synthesis of acrylic acid from ethylene and $\mathrm{CO}_{2}$. (c) Ethylene glycol synthesis via reductive coupling of $\mathrm{CO}_{2}$. (d) Reductive methylation of benzene using $\mathrm{H}_{2}$ and $\mathrm{CO}_{2}$. 
hydrogenation of $\mathrm{CO}_{2}$ to produce this chemical in bulk scale might be a prime example for selective reductive coupling using carbon dioxide and could substitute its traditional synthesis from ethylene oxide (Fig. 8, equation c). As a final example, the reductive methylation of more widely available arenes, for example, benzene (from biogas), to generate toluene and xylenes is also highly interesting (Fig. 8, equation d).

Above all, it is anticipated that more research groups will join this very promising but also challenging field to discover a number of applied organic synthesis approaches using $\mathrm{CO}_{2}$ as the $\mathrm{C} 1$ building block, in which the employment of less-active substrates, reduced waste generation and milder reaction conditions should be addressed.

\section{References}

1. Schierbaum, F. in Carbon Dioxide as Chemical Feedstock Vol. 62 (ed. Aresta, M.) (Wiley-VCH, 2010).

2. Mikkelsen, M., Jorgensen, M. \& Krebs, F. C. The teraton challenge. A review of fixation and transformation of carbon dioxide. Energy Environ. Sci. 3, 43-81 (2010).

3. Sakakura, T., Choi, J.-C. \& Yasuda, H. Transformation of carbon dioxide. Chem. Rev. 107, 2365-2387 (2007).

4. Maeda, C., Miyazaki, Y. \& Ema, T. Recent progress in catalytic conversions of carbon dioxide. Catal. Sci. Technol. 4, 1482-1497 (2014).

5. Federsel, C., Jackstell, R. \& Beller, M. State-of-the-art catalysts for hydrogenation of carbon dioxide. Angew. Chem. Int. Ed. 49, 6254-6257 (2010).

6. Jian, G., Bing, Y. \& Liang-Nian, H. in Production and Purification of Ultraclean Transportation Fuels. ACS Symposium Series Vol. 1088, Ch. 8, 143-174 (American Chemical Society, 2011).

7. Wang, W., Wang, S., Ma, X. \& Gong, J. Recent advances in catalytic hydrogenation of carbon dioxide. Chem. Soc. Rev. 40, 3703-3727 (2011).

8. Coates, G. W. \& Moore, D. R. Discrete metal-based catalysts for the copolymerization of $\mathrm{CO}_{2}$ and epoxides: discovery, reactivity, optimization, and mechanism. Angew. Chem. Int. Ed. 43, 6618-6639 (2004).

9. Sakakura, T. \& Kohno, K. The synthesis of organic carbonates from carbon dioxide. Chem. Commun. 11, 1312-1330 (2009).

10. Clements, J. H. Reactive applications of cyclic alkylene carbonates. Ind. Eng. Chem. Res. 42, 663-674 (2003).

11. Martin, R. \& Kleij, A. W. Myth or reality? Fixation of carbon dioxide into complex organic matter under mild conditions. ChemSusChem 4, 1259-1263 (2011).

12. Aida, T. \& Inoue, S. Activation of carbon dioxide with aluminum porphyrin and reaction with epoxide. Studies on (tetraphenylporphinato)aluminum alkoxide having a long oxyalkylene chain as the alkoxide group. J. Am. Chem. Soc. 105, 1304-1309 (1983).

13. Jin, L. et al. Metal porphyrin/phenyltrimethylammonium tribromide: high efficient catalysts for coupling reaction of $\mathrm{CO}_{2}$ and epoxides. J. Mol. Catal. A Chem. 261, 262-266 (2007).

14. Ema, T., Miyazaki, Y., Koyama, S., Yano, Y. \& Sakai, T. A bifunctional catalyst for carbon dioxide fixation: cooperative double activation of epoxides for the synthesis of cyclic carbonates. Chem. Commun. 48, 4489-4491 (2012).

15. Paddock, R. L. \& Nguyen, S. T. Chemical $\mathrm{CO}_{2}$ fixation: $\mathrm{Cr}(\mathrm{III})$ salen complexes as highly efficient catalysts for the coupling of $\mathrm{CO}_{2}$ and epoxides. $J$. Am. Chem. Soc. 123, 11498-11499 (2001).

16. Decortes, A. \& Kleij, A. W. Ambient fixation of carbon dioxide using a ZnII salphen catalyst. Chem CatChem 3, 831-834 (2011).

17. North, M. \& Pasquale, R. Mechanism of cyclic carbonate synthesis from epoxides and $\mathrm{CO}_{2}$. Angew. Chem. Int. Ed. 48, 2946-2948 (2009).

18. Whiteoak, C. J. et al. A powerful aluminum catalyst for the synthesis of highly functional organic carbonates. J. Am. Chem. Soc. 135, 1228-1231 (2013).

19. Li, F., Xiao, L., Xia, C. \& Hu, B. Chemical fixation of $\mathrm{CO}_{2}$ with highly efficient $\mathrm{ZnCl}_{2} /[\mathrm{BMIm}] \mathrm{Br}$ catalyst system. Tetrahedron Lett. 45, 8307-8310 (2004).

20. Liang, S. et al. Highly efficient synthesis of cyclic carbonates from $\mathrm{CO}_{2}$ and epoxides over cellulose/KI. Chem. Commun. 47, 2131-2133 (2011).

21. Cheng, M., Lobkovsky, E. B. \& Coates, G. W. Catalytic reactions involving C1 feedstocks: new high-activity $\mathrm{Zn}(\mathrm{II})$-based catalysts for the alternating copolymerization of carbon dioxide and epoxides. J. Am. Chem. Soc. 120, 11018-11019 (1998).

22. Moore, D. R., Cheng, M., Lobkovsky, E. B. \& Coates, G. W. Mechanism of the alternating copolymerization of epoxides and $\mathrm{co}_{2}$ using $\beta$-diiminate zinc catalysts: evidence for a bimetallic epoxide enchainment. J. Am. Chem. Soc. 125, 11911-11924 (2003).

23. Kember, M. R., Knight, P. D., Reung, P. T. R. \& Williams, C. K. Highly active dizinc catalyst for the copolymerization of carbon dioxide and cyclohexene oxide at one atmosphere pressure. Angew. Chem. Int. Ed. 48, 931-933 (2009).
24. Nakano, K., Kobayashi, K., Ohkawara, T., Imoto, H. \& Nozaki, K. Copolymerization of epoxides with carbon dioxide catalyzed by iron-corrole complexes: synthesis of a crystalline copolymer. J. Am. Chem. Soc. 135, $8456-8459$ (2013)

25. Nozaki, K., Nakano, K. \& Hiyama, T. Optically active polycarbonates: asymmetric alternating copolymerization of cyclohexene oxide and carbon dioxide. J. Am. Chem. Soc. 121, 11008-11009 (1999).

26. Hua, Y.-Z. et al. Highly enantioselective catalytic system for asymmetric copolymerization of carbon dioxide and cyclohexene oxide. Chem. Eur. J. 20, 12394-12398 (2014).

27. Martin, C. et al. Easily accessible bifunctional Zn(salpyr) catalysts for the formation of organic carbonates. Catal. Sci. Technol. 4, 1615-1621 (2014).

28. Lim, C.-H., Holder, A. M., Hynes, J. T. \& Musgrave, C. B. Roles of the Lewis acid and base in the chemical reduction of $\mathrm{CO}_{2}$ catalyzed by frustrated Lewis pairs. Inorg. Chem. 52, 10062-10066 (2013).

29. Tlili, A., Frogneux, X., Blondiaux, E. \& Cantat, T. Creating added value with a waste: methylation of amines with $\mathrm{CO}_{2}$ and $\mathrm{H}_{2}$. Angew. Chem. Int. Ed. 53, 2543-2545 (2014)

30. Das Neves Gomes, C. et al. A diagonal approach to chemical recycling of carbon dioxide: organocatalytic transformation for the reductive functionalization of $\mathrm{CO}_{2}$. Angew. Chem. Int. Ed. 51, 187-190 (2012). A pioneering example of diagonal transformations for $\mathrm{CO}_{2}$ recycling, which combine both reduction of $\mathrm{CO}_{2}$ and formation of $\mathrm{C}-\mathrm{C}, \mathrm{C}-\mathrm{N}$, or $\mathrm{C}-\mathrm{O}$ bonds with $\mathrm{CO}_{2}$.

31. Jacquet, O., Das Neves Gomes, C., Ephritikhine, M. \& Cantat, T. Recycling of carbon and silicon wastes: room temperature formylation of $\mathrm{N}-\mathrm{H}$ bonds using carbon dioxide and polymethylhydrosiloxane. J. Am. Chem. Soc. 134, 2934-2937 (2012).

32. Li, Y., Fang, X., Junge, K. \& Beller, M. A general catalytic methylation of amines using carbon dioxide. Angew. Chem. Int. Ed. 52, 9568-9571 (2013)

33. Jacquet, O., Frogneux, X., Das Neves Gomes, C. \& Cantat, T. $\mathrm{CO}_{2}$ as a C1-building block for the catalytic methylation of amines. Chem. Sci. 4, 2127-2131 (2013).

34. Beydoun, K., vom Stein, T., Klankermayer, J. \& Leitner, W. Rutheniumcatalyzed direct methylation of primary and secondary aromatic amines using carbon dioxide and molecular hydrogen. Angew. Chem. Int. Ed. 52, 9554-9557 (2013).

35. Li, Y., Sorribes, I., Yan, T., Junge, K. \& Beller, M. Selective methylation of amines with carbon dioxide and $\mathrm{H}_{2}$. Angew. Chem. Int. Ed. 52, 12156-12160 (2013).

36. Cui, X., Dai, X., Zhang, Y., Deng, Y. \& Shi, F. Methylation of amines, nitrobenzenes and aromatic nitriles with carbon dioxide and molecular hydrogen. Chem. Sci. 5, 649-655 (2014).

37. Aresta, M., Nobile, C. F., Albano, V. G., Forni, E. \& Manassero, M. New nickel-carbon dioxide complex. Synthesis, properties, and crystallographic characterization of (carbon dioxide)bis(tricyclohexylphosphine)nickel. J. Chem. Soc. Chem. Commun. 636-637 (1975).

We consider this paper pivotal in developing the strategy of $\mathrm{CO}_{2}$ acitivation via oxidative cyclometallation; the first metallacycle complex from $\mathrm{CO}_{2}$ was reported.

38. Hoberg, H., Peres, Y., Krueger, C. \& Tsay, Y. H. A 1-oxa-2-nickela-5cyclopentanone from ethene and carbon dioxide; preparation, structure and reactivity. Angew. Chem. 99, 799-800 (1987).

39. Burkhart, G. \& Hoberg, H. Oxanickelacyclopentene derivatives from nickel(0), carbon dioxide, and alkynes. Angew. Chem. 94, 75 (1982).

40. Takimoto, M. \& Mori, M. Cross-coupling reaction of oxo- $\pi$-allylnickel complex generated from 1,3-diene under an atmosphere of carbon dioxide. J. Am. Chem. Soc. 123, 2895-2896 (2001).

41. Louie, J., Gibby, J. E., Farnworth, M. V. \& Tekavec, T. N. Efficient nickelcatalyzed $[2+2+2]$ cycloaddition of $\mathrm{CO}_{2}$ and diynes. J. Am. Chem. Soc. 124, 15188-15189 (2002).

42. Takimoto, M. \& Mori, M. Novel catalytic $\mathrm{CO}_{2}$ incorporation reaction: nickel-catalyzed regio- and stereoselective ring-closing carboxylation of bis-1,3-dienes. J. Am. Chem. Soc. 124, 10008-10009 (2002).

43. Takimoto, M., Nakamura, Y., Kimura, K. \& Mori, M. Highly enantioselective catalytic carbon dioxide incorporation reaction: nickel-catalyzed asymmetric carboxylative cyclization of bis-1,3-dienes. J. Am. Chem. Soc. 126, 5956-5957 (2004).

44. Behr, A. \& Juszak, K.-D. Palladium-catalyzed reaction of butadiene and carbon dioxide. J. Organomet. Chem. 255, 263-268 (1983).

45. Yeung, C. S. \& Dong, V. M. Beyond Aresta's complex: Ni- and Pdcatalyzed organozinc coupling with $\mathrm{CO}_{2}$. J. Am. Chem. Soc. 130, 7826-7827 (2008).

46. Huang, K., Sun, C.-L. \& Shi, Z.-J. Transition metal-catalyzed C-C bond formation through the fixation of carbon dioxide. Chem. Soc. Rev. 40, 2435-2452 (2011).

A seminal introduction to $\mathrm{CO}_{2}$ valorization reactions with $\mathrm{C}-\mathrm{C}$ bond formation and its application in organic synthesis. 
47. Behr, A. Carbon dioxide as an alternative C1-building block: activation by transition metal complexes. Angew. Chem. 100, 681-698 (1988).

48. de Meijere, A. \& Diederich, F. (eds) Metal-Catalyzed Cross-Coupling Reactions 2nd, Completely Revised and Enlarged Edition, Vol. 2 (Wiley-VCH, 2004).

49. Correa, A. \& Martin, R. Metal-catalyzed carboxylation of organometallic reagents with carbon dioxide. Angew. Chem. Int. Ed. 48, 6201-6204 (2009)

50. Ackermann, L. Transition-metal-catalyzed carboxylation of C-H bonds. Angew. Chem. Int. Ed. 50, 3842-3844 (2011).

51. Dalton, D. M. \& Rovis, T. C-H carboxylation takes gold. Nat. Chem. 2 , 710-711 (2010).

52. Zhang, Y.-G. \& Riduan, S. N. Catalytic hydrocarboxylation of alkenes and alkynes with $\mathrm{CO}_{2}$. Angew. Chem. Int. Ed. 50, 6210-6212 (2011).

53. Ukai, K., Aoki, M., Takaya, J. \& Iwasawa, N. Rhodium(I)-catalyzed carboxylation of aryl- and alkenylboronic esters with $\mathrm{CO}_{2}$. J. Am. Chem. Soc. 128, 8706-8707 (2006)

The authors reported the first catalytic carboxylation reaction of weak carbon nucleophiles with $\mathrm{CO}_{2}$

54. Takaya, J., Tadami, S., Ukai, K. \& Iwasawa, N. Copper(I)-catalyzed carboxylation of aryl- and alkenylboronic esters. Org. Lett. 10, 2697-2700 (2008).

55. Ohishi, T., Nishiura, M. \& Hou, Z. Carboxylation of organoboronic esters catalyzed by N-heterocyclic carbene copper(I) complexes. Angew. Chem. Int. Ed. 47, 5792-5795 (2008).

56. Ohmiya, H., Tanabe, M. \& Sawamura, M. Copper-catalyzed carboxylation of alkylboranes with carbon dioxide: formal reductive carboxylation of terminal alkenes. Org. Lett. 13, 1086-1088 (2011).

57. Ohishi, T., Zhang, L., Nishiura, M. \& Hou, Z. Carboxylation of alkylboranes by N-heterocyclic carbene copper catalysts: synthesis of carboxylic acids from terminal alkenes and carbon dioxide. Angew. Chem. Int. Ed. 50, 8114-8117 (2011).

58. Zhang, X. et al. Silver(I)-catalyzed carboxylation of arylboronic esters with $\mathrm{CO}_{2}$. Chem. Commun. 48, 6292-6294 (2012).

59. Zhang, X., Zhang, W.-Z., Ren, X., Zhang, L.-L. \& Lu, X.-B. Ligand-free Ag(I)catalyzed carboxylation of terminal alkynes with $\mathrm{CO}_{2}$. Org. Lett. 13, 2402-2405 (2011).

60. Yu, D. \& Zhang, Y. The direct carboxylation of terminal alkynes with carbon dioxide. Green Chem. 13, 1275-1279 (2011).

61. Yu, D. \& Zhang, Y. Copper- and copper-N-heterocyclic carbene-catalyzed C $\mathrm{H}$ activating carboxylation of terminal alkynes with $\mathrm{CO}_{2}$ at ambient conditions. Proc. Natl Acad. Sci. USA 107, $20184-20189$ (2010).

62. Goossen, L. J., Rodriguez, N., Manjolinho, F. \& Lange, P. P. Synthesis of propiolic acids via copper-catalyzed insertion of carbon dioxide into the $\mathrm{C}-\mathrm{H}$ bond of terminal alkynes. Adv. Synth. Catal. 352, 2913-2917 (2010).

63. Zhang, L., Cheng, J., Ohishi, T. \& Hou, Z. Copper-catalyzed direct carboxylation of $\mathrm{C}-\mathrm{H}$ bonds with carbon dioxide. Angew. Chem. Int. Ed. 49, 8670-8673 (2010).

64. Vechorkin, O., Hirt, N. \& Hu, X. Carbon dioxide as the $\mathrm{C} 1$ source for direct C-H functionalization of aromatic heterocycles. Org. Lett. 12, 3567-3569 (2010).

65. Boogaerts, I. I. F. \& Nolan, S. P. Carboxylation of C-H bonds using $\mathrm{N}$-heterocyclic carbene gold(I) complexes. J. Am. Chem. Soc. 132, 8858-8859 (2010).

66. Boogaerts, I. I. F., Fortman, G. C., Furst, M. R. L., Cazin, C. S. J. \& Nolan, S. P. Carboxylation of N-H/C-H bonds using N-heterocyclic carbene copper(I) complexes. Angew. Chem. Int. Ed. 49, 8674-8677 (2010).

67. Sasano, K., Takaya, J. \& Iwasawa, N. Palladium(II)-catalyzed direct carboxylation of alkenyl C-H bonds with $\mathrm{CO}_{2}$. J. Am. Chem. Soc. 135, 10954-10957 (2013).

68. Mizuno, H., Takaya, J. \& Iwasawa, N. Rhodium(I)-catalyzed direct carboxylation of arenes with $\mathrm{CO}_{2}$ via chelation-assisted $\mathrm{C}-\mathrm{H}$ bond activation. J. Am. Chem. Soc. 133, 1251-1253 (2011).

69. Correa, A. \& Martin, R. Palladium-catalyzed direct carboxylation of aryl bromides with carbon dioxide. J. Am. Chem. Soc. 131, 15974-15975 (2009).

70. Fujihara, T., Nogi, K., Xu, T., Terao, J. \& Tsuji, Y. Nickel-catalyzed carboxylation of aryl and vinyl chlorides employing carbon dioxide. J. Am. Chem. Soc. 134, 9106-9109 (2012).

71. León, T., Correa, A. \& Martin, R. Ni-catalyzed direct carboxylation of benzyl halides with $\mathrm{CO}_{2}$. J. Am. Chem. Soc. 135, 1221-1224 (2013).

72. Correa, A., León, T. \& Martin, R. Ni-catalyzed carboxylation of $\mathrm{C}(\mathrm{sp} 2)-$ and $\mathrm{C}(\mathrm{sp} 3)-\mathrm{O}$ bonds with $\mathrm{CO}_{2}$. J. Am. Chem. Soc. 136, 1062-1069 (2013).

73. Takaya, J. \& Iwasawa, N. Hydrocarboxylation of allenes with $\mathrm{CO}_{2}$ catalyzed by silyl pincer-type palladium complex. J. Am. Chem. Soc. 130, 15254-15255 (2008).

74. Williams, C. M., Johnson, J. B. \& Rovis, T. Nickel-catalyzed reductive carboxylation of styrenes using $\mathrm{CO}_{2}$. J. Am. Chem. Soc. 130, 14936-14937 (2008).
75. Li, S., Yuan, W. \& Ma, S. Highly regio- and stereoselective three-component nickel-catalyzed syn-hydrocarboxylation of alkynes with diethyl zinc and carbon dioxide. Angew. Chem. Int. Ed. 50, 2578-2582 (2011).

76. Fujihara, T., Xu, T., Semba, K., Terao, J. \& Tsuji, Y. Copper-catalyzed hydrocarboxylation of alkynes using carbon dioxide and hydrosilanes. Angew. Chem. Int. Ed. 50, 523-527 (2011).

77. Zhang, L., Cheng, J., Carry, B. \& Hou, Z. Catalytic boracarboxylation of alkynes with diborane and carbon dioxide by an N-heterocyclic carbene copper catalyst. J. Am. Chem. Soc. 134, 14314-14317 (2012).

78. Franke, R., Selent, D. \& Boerner, A. Applied hydroformylation. Chem. Rev. 112, 5675-5732 (2012).

79. Eastham, G. R., Waugh, M., Pringle, P. \& Turner, T. P. W. Process for the carbonylation of ethylenically unsaturated compounds, bidentate carbonylation ligands and catalyst systems incorporating such ligands WO2011083305 (2011)

80. Tominaga, K.-I. \& Sasaki, Y. Ruthenium complex-catalyzed hydroformylation of alkenes with carbon dioxide. Catal. Commun. 1, 1-3 (2000).

This work creats the benchmark for the directly carbonylation of alkenes with $\mathrm{CO}_{2}$, in which $\mathrm{CO}$ is in-situ generated from $\mathrm{CO}_{2}$ hydrogenation (reverse water gas shift process).

81. Liu, Q. et al. Development of a ruthenium/phosphite catalyst system for domino hydroformylation-reduction of olefins with carbon dioxide. Chem. Eur. J. 20, 6888-6894 (2014).

82. Ostapowicz, T. G., Schmitz, M., Krystof, M., Klankermayer, J. \& Leitner, W. Carbon dioxide as a $\mathrm{C} 1$ building block for the formation of carboxylic acids by formal catalytic hydrocarboxylation. Angew. Chem. Int. Ed. 52, 12119-12123 (2013).

83. Nielsen, M. et al. Low-temperature aqueous-phase methanol dehydrogenation to hydrogen and carbon dioxide. Nature 495, 85-89 (2013).

84. Ziebart, C. et al. Well-defined iron catalyst for improved hydrogenation of carbon dioxide and bicarbonate. J. Am. Chem. Soc. 134, 20701-20704 (2012).

85. Wu, L., Liu, Q., Fleischer, I., Jackstell, R. \& Beller, M. Ruthenium-catalysed alkoxycarbonylation of alkenes with carbon dioxide. Nat. Commun. 5, 3091 (2014).

This paper presents the first catalytic system for the $\mathrm{CO}_{2}$ reduction to $\mathrm{CO}$ using alcohols as the reductant and also its application in the alkoxycarbonylation of alkenes with $\mathrm{CO}_{2}$

86. Lescot, C. et al. Efficient fluoride-catalyzed conversion of $\mathrm{CO}_{2}$ to $\mathrm{CO}$ at room temperature. J. Am. Chem. Soc. 136, 6142-6147 (2014).

87. Reithmeier, R., Bruckmeier, C. \& Rieger, B. Conversion of $\mathrm{CO}_{2}$ via visible light promoted homogeneous redox catalysis. Catalysts 2, 544-571 (2012).

88. Lehn, J.-M. \& Ziessel, R. Photochemical generation of carbon monoxide and hydrogen by reduction of carbon dioxide and water under visible light irradiation. Proc. Natl Acad. Sci. USA 79, 701-704 (1982)

89. Hawecker, J., Lehn, J.-M. \& Ziessel, R. Efficient photochemical reduction of $\mathrm{CO}_{2}$ to $\mathrm{CO}$ by visible light irradiation of systems containing $\operatorname{Re}($ bipy $)(\mathrm{CO})_{3} \mathrm{X}$ or $\mathrm{Ru}$ (bipy) ${ }_{3}^{2+}-\mathrm{Co}^{2+}$ combinations as homogeneous catalysts. J. Chem. Soc. Chem. Commun. 536-538 (1983).

90. Hawecker, J., Lehn, J.-M. \& Ziessel, R. Photochemical and electrochemical reduction of carbon dioxide to carbon monoxide mediated by $\left(2,2^{\prime}-\right.$ bipyridine)tricarbonylchlororhenium(I) and related complexes as homogeneous catalysts. Helv. Chim. Acta 69, 1990-2012 (1986).

91. Hayashi, Y., Kita, S., Brunschwig, B. S. \& Fujita, E. Involvement of a binuclear species with the $\mathrm{Re}-\mathrm{C}(\mathrm{O}) \mathrm{O}-\mathrm{Re}$ moiety in $\mathrm{CO}_{2}$ reduction catalyzed by tricarbonyl rhenium(I) complexes with diimine ligands: strikingly slow formation of the $\mathrm{Re}-\mathrm{Re}$ and $\mathrm{Re}-\mathrm{C}(\mathrm{O}) \mathrm{O}-\mathrm{Re}$ species from $\mathrm{Re}(\mathrm{dmb})(\mathrm{CO})_{3} \mathrm{~S}$ ( $\mathrm{dmb}=4,4^{\prime}$-dimethyl-2,2'-bipyridine, $\mathrm{S}=$ solvent). J. Am. Chem. Soc. 125, 11976-11987 (2003)

92. Takeda, H., Koike, K., Inoue, H. \& Ishitani, O. Development of an efficient photocatalytic system for $\mathrm{CO}_{2}$ reduction using rhenium(I) complexes based on mechanistic studies. J. Am. Chem. Soc. 130, 2023-2031 (2008).

93. Bian, Z.-Y. et al. A novel tripodal ligand, Tris $\left[\left(4^{\prime}\right.\right.$-methyl-2,2'-bipyridyl-4yl)methyl]carbinol and its trinuclear RuII/ReI mixed-metal complexes: synthesis, emission properties, and photocatalytic $\mathrm{CO}_{2}$ reduction. Inorg. Chem. 47, 10801-10803 (2008).

94. Gholamkhass, B. et al. Architecture of supramolecular metal complexes for photocatalytic $\mathrm{CO}_{2}$ reduction: ruthenium - rhenium $\mathrm{Bi}$ - and tetranuclear complexes. Inorg. Chem. 44, 2326-2336 (2005).

95. Sato, S., Morikawa, T., Kajino, T. \& Ishitani, O. A highly efficient mononuclear iridium complex photocatalyst for $\mathrm{CO}_{2}$ reduction under visible light. Angew. Chem. Int. Ed. 52, 988-992 (2013).

96. Ettedgui, J., Diskin-Posner, Y., Weiner, L. \& Neumann, R. Photoreduction of carbon dioxide to carbon monoxide with hydrogen catalyzed by a rhenium(I) phenanthroline-polyoxometalate hybrid complex. J. Am. Chem. Soc. 133, 188-190 (2011) 
97. Nakano, R., Ito, S. \& Nozaki, K. Copolymerization of carbon dioxide and butadiene via a lactone intermediate. Nat. Chem. 6, 325-331 (2014).

98. Wu, L., Fleischer, I., Jackstell, R. \& Beller, M. Efficient and regioselective ruthenium-catalyzed hydro-aminomethylation of olefins. J. Am. Chem. Soc. 135, 3989-3996 (2013).

99. Wu, L. et al. Ruthenium-catalyzed hydroformylation/reduction of olefins to alcohols: extending the scope to internal alkenes. J. Am. Chem. Soc. 135, 14306-14312 (2013).

100. Lejkowski, M. L. et al. The first catalytic synthesis of an acrylate from $\mathrm{CO}_{2}$ and an alkene-A rational approach. Chem. Eur. J. 18, 14017-14025 (2012).

\section{Acknowledgements}

We thank the State of Mecklenburg-Vorpommern and the BMBF for financial support. Q.L. also appreciates the grant from Alexander von Humboldt Foundation.

\section{Additional information}

Competing financial interests: The authors declare no competing financial interests.

Reprints and permission information is available online at http://npg.nature.com/ reprintsandpermissions/

How to cite this article: Liu, Q. et al. Using carbon dioxide as a building block in organic synthesis. Nat. Commun. 6:5933 doi: 10.1038/ncomms6933 (2015). 